\author{
Department of Nephrology and Rheumatology \\ (Prof. Dr. med. G. A. Müller) \\ Center for Internal Medicine
}

Faculty of Medicine at the Georg-August-University Göttingen

\title{
Peroxisomes and Kidney Damage
}

In partial fulfilment of the requirements for the degree

"Doctor of medicine (Dr. med.)"

at the Faculty of Medicine

at the Georg-August-University Göttingen

submitted by

\section{Radovan Vasko}

born in

Sala

Göttingen, 2014 
Dean: $\quad$ Prof. Dr. rer. nat. H. K. Kroemer

Referee: $\quad$ Prof. Dr. rer. nat. H. Dihazi

Referee: $\quad$ Prof. Dr. rer. nat. M. Thumm

Date of examination: 16.12.2014 


\section{Content}

\section{List of Abbreviations}

1. Introduction 1

1.1 Peroxisomal biogenesis 2

1.2 Peroxisomal fatty acid oxidation 6

1.3 Peroxisomal metabolism of reactive oxygen species 8

1.4 Peroxisomal disorders 9

1.5 Peroxisomal homeostasis and autophagy 12

$\begin{array}{lll}1.6 & \text { Peroxisomes and kidney } & 15\end{array}$

2. Peroxisomes and pexophagy in LPS-induced acute kidney injury 18

2.1 Acute kidney injury in mice with impaired pexophagy 18

$\begin{array}{ll}2.2 & \text { Endothelium, sepsis and cytokine storm }\end{array}$

2.3 Renal peroxisomes in endotoxic stress 23

$2.4 \quad$ Endothelial peroxisomes in endotoxic stress 25

2.5 Peroxisomal import and LPS 29

2.6 Lysosomal dysfunction and reactive oxygen species 31

2.7 Metabolic-oxidative imbalance in lysosomal dysfunction 32

$\begin{array}{ll}\text { 3. Discussion } & 37\end{array}$

4. References $\quad 42$ 


\section{Thesis publication}

Vasko R, Ratliff BB, Bohr S, Nadel E, Chen J, Xavier S, Chander P, Goligorsky MS (2013):

Endothelial Peroxisomal Dysfunction and Impaired Pexophagy Promotes Oxidative Damage in Lipopolysaccharide-Induced Acute Kidney Injury. Antioxid Redox Signal 19 (3):211-230. IF 7.2

\section{Additional topic publications}

Vasko R, Goligorsky MS (2013): Dysfunctional lysosomal autophagy leads to peroxisomal oxidative burnout and damage during endotoxin-induced stress. Autophagy 2(3):442-444. IF 12.0

Ratliff BB, Rabadi MM, Vasko R, Yasuda K, Goligorsky MS (2013): Messengers without borders: mediators of systemic inflammatory response in AKI. J Am Soc Nephrol 244(4):529-536. IF 8.9 


\section{List of Abbreviations}

\begin{tabular}{|c|c|}
\hline AKI & acute kidney injury \\
\hline ATP & adenosine triphosphate \\
\hline $\mathrm{ABC}$ & ATP-binding cassette \\
\hline ACOX & acyl-CoA-oxidase \\
\hline CMV & cytomegalovirus \\
\hline DNA & deoxyribonucleic acid \\
\hline FAO & fatty acids oxidation \\
\hline FFA & free fatty acids \\
\hline GFP & green fluorescent protein \\
\hline G-CSF & granulocyte colony-stimulating factor \\
\hline GM-CSF & granulocyte-macrophage colony-stimulating factor \\
\hline $\mathrm{H}_{2} \mathrm{O}_{2}$ & hydrogen peroxide \\
\hline IL & interleukin \\
\hline TNF $\alpha$ & tumor necrosis factor alpha \\
\hline LC3 & microtubule-associated protein $1 \mathrm{~A} / 1 \mathrm{~B}$-light chain 3 \\
\hline LIR & LC3-interacting region \\
\hline LPS & lipopolysaccharide \\
\hline MCP-1 & monocyte chemoattractant protein-1 \\
\hline P62 & p62=sequestosome- 1 \\
\hline Pex & peroxin \\
\hline
\end{tabular}


PGC-1 $\alpha$ peroxisome proliferator-activated receptor-gamma coactivator 1 alpha

PMP70 peroxisomal membrane protein 70

$\operatorname{PPAR} \alpha \quad$ peroxisome proliferator-activated receptor alpha

PPRE peroxisome proliferator response element

PTS peroxisomal targeting signal

ROS reactive oxygen species

UBD ubiquitin-binding domain

VLCFA very long chain fatty acids 


\section{Introduction}

In 1954, in early electron microscopy studies of murine proximal tubules, Swedish doctoral student Johannes Rhodin described in his thesis small spherical organelles, and named them "microbodies" (Rhodin 1954). These organelles were later chemically characterized by Belgian biochemist Christian de Duve in extracts from rat liver, and due to the high content of enzymes generating and decomposing hydrogen peroxide, the microscopic term "microbodies" was replaced by "peroxisomes" (De Duve and Baudhuin 1966). In recent years, peroxisomes, early designated as fossil organelles, have attracted more and more attention and became recognized as indispensable actors in cellular pathways under physiological conditions and in disease.

Mammalian peroxisomes are $0,1-0,5 \mu \mathrm{M}$ sized single membrane organelles present in virtually all cells, with the highest abundance in the liver and the kidney. They possess a comprehensive apparatus of more than 50 enzymes and accomplish a multitude of diverse biological functions. The two most important metabolic tasks performed by peroxisomes are the oxidation of fatty acids and generation/decomposition of hydrogen peroxide $\left(\mathrm{H}_{2} \mathrm{O}_{2}\right)$ (Platta and Erdmann 2007). Peroxisomes do not have genome and therefore have to import their proteins from cytosol. Peroxisomes contain their specific proteins named peroxins, which are encoded by Pex genes (Purdue and Lazarow 2001). Peroxins participate in peroxisomal proliferation, division, transport and import of substrates from cytosol, organelle movement, and also in degradation of aged or damaged peroxisomes. More than 30 different peroxins have been discovered and described so far. Peroxins are components of the peroxisomal matrix or peroxisomal membrane, and some of them travel between peroxisomes and cytosol. 
Contribution of peroxisomes to the pathogenesis of human diseases has been widely accepted by the discovery of disorders caused by defective peroxisomal biogenesis and/or defects in specific peroxisomal enzymes. Accumulating evidence indicates that peroxisomes are actively involved in apoptosis (Hasegawa et al. 2010), inflammation (Zmijewski et al. 2009), immunity (Dixit et al. 2010), aging and age-related diseases, such as diabetes or cancer (Fransen et al. 2013).

\subsection{Peroxisomal biogenesis}

The evolutionary origin of peroxisomes is still a matter of discussion (Gabaldon 2010). According to one of the current theories, evolution of peroxisomes was a consequence of raising oxygen levels in the archaic atmosphere (De Duve 2007). Peroxisomes are not remnants of endosymbiotic organisms, like mitochondria, but have developed from endomembrane compartment structure, probably from specialized parts of endoplasmic reticulum (Schluter et al. 2006). Besides the controversies in the evolutionary origin of peroxisomes, the origin of this organelle at subcellular level (biogenesis) is also not completely clear. The classical model of peroxisomal biogenesis states that new organelles arise from preexisting peroxisomes through growth and division (Lazarow and Fujiki 1985). However, according to recent studies, peroxisomes can also be generated de novo in a maturation process utilizing pre-peroxisomal vesicles budding from endoplasmic reticulum (Nuttall et al. 2011). Peroxisomes have a protein rich fluid matrix that is enclosed by a single membrane. As mentioned above, peroxisomes do not possess DNA and must import their matrix and membrane proteins from cytoplasm. Peroxisomal proteins are synthesized on free cytoplasmic ribosomes and subsequently transported to peroxisomes with the help of specific transporters that recognize distinct short amino acid 
sequences of these proteins, so-called peroxisomal targeting signals (PTS) (Brink and Danpure 2001). Peroxisomal targeting signal 1 (PTS1), a serine-lysine-leucine tripeptide sequence is present at carboxy termini of proteins, and represents the main PTS present on peroxisomal matrix proteins. A second type of signal peptide, peroxisomal targeting signal 2 (PTS2), has been identified as an 11-amino acid sequence at amino termini of a smaller fraction of peroxisomal matrix proteins. Peroxisomal membrane proteins have peroxisomal membrane targeting signal (mPTS) that has not been precisely characterized as the PTS1 and PTS2 on peroxisomal matrix proteins. The exact process of recognition, binding, transportation and intraorganellar import of peroxisomal proteins is far too complex to discuss in detail here. For more information on peroxisomal protein import, please refer to review by Rucktäschel and colleagues (Rucktaschel et al. 2011). In this introduction, I will briefly describe important features and pathways participating in peroxisomal import that are relevant for understanding of mechanisms studied here and discussed further in the text. Basically, the import of peroxisomal matrix proteins can be defined in four consecutive steps (Islinger et al. 2012): (1) Individual matrix protein (cargo) in cytoplasm is recognized via PTS and bound by the corresponding cytosolic receptor. PTS1 containing proteins are recognized by peroxin 5 (Pex5), PTS2 containing proteins by peroxin 7 (Pex7). These cargo-receptor complexes are transported to peroxisomes where they (2) dock to Pex14 at the peroxisomal membrane. After docking, the cargo-receptor complex is integrated and (3) further translocated across the peroxisomal membrane. (4) After releasing of the protein in peroxisomal matrix, Pex 5 or Pex7 receptors are exported back to cytosol (in an energy-dependent process), where they become available for new import cycle (Figure 1). The exact molecular basis of protein translocation across the peroxisomal membrane is still not fully understood. 


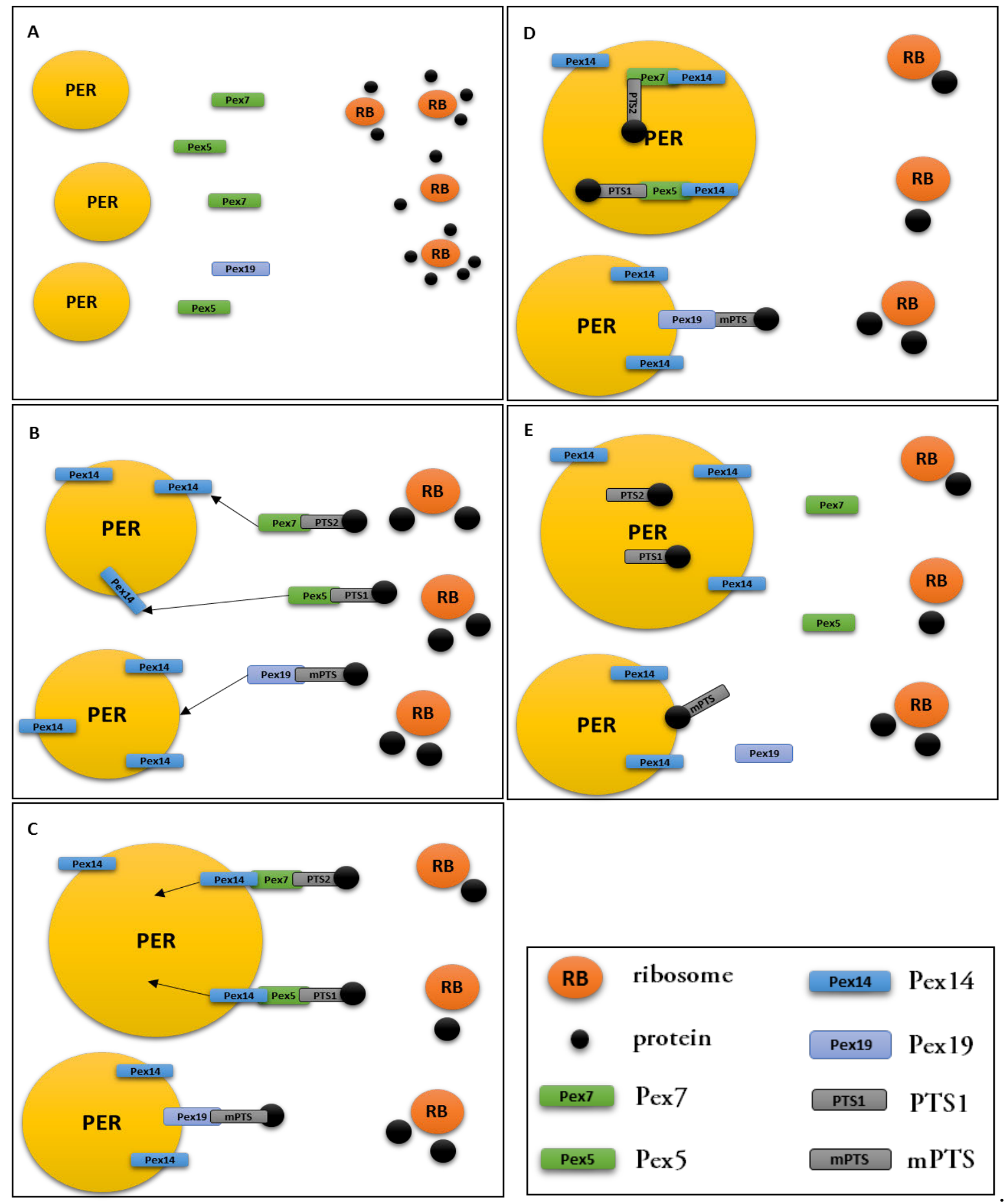

Figure 1. Peroxisomal protein import. (A-B) Peroxisomal matrix proteins synthesized on cytosolic ribosomes (RB) are recognized and bound by cytosolic receptors Pex5 or Pex7 via PTS1 or PTS2 respectively. (C) Protein-receptor complexes are transported to peroxisomes (PER) and dock to Pex14 at the peroxisomal membrane. (D) Subsequently, protein-receptor complexes are translocated and proteins released into peroxisomal matrix. (E) Pex5 and Pex7 are recycled and exported back to cytosol. Peroxisomal membrane proteins are recognized via mPTS by Pex19, and after transportation to peroxisomes, integrated into peroxisomal membrane. 
One of the proposed models follows the hypothesis of a transient membrane pore that is generated through interaction of PTS receptor Pex5 and the docking peroxin Pex14 (Ma et al. 2009). Peroxisomal membrane proteins are recognized via mPTS by cytoplasmic receptor Pex19. After transportation and docking to peroxisomes, they are incorporated into peroxisomal membrane (Rottensteiner et al. 2004). A small group of peroxisomal membrane proteins lack the mPTS sequence specifically recognized by Pex 19 , and is probably transported to peroxisomes via endoplasmic reticulum by a vesicle-mediated mechanism (Ma et al. 2011). Peroxisomes are highly active organelles. For accurate functioning of the multitude of metabolic processes inside peroxisomes, they need to import numerous metabolic substrates and cofactors required by their enzymes. Peroxisomal membrane appears to be permeable to small metabolites up to a size of 400-500 Dalton. Larger molecules, such as fatty acids, and a lot of intermediate molecules, as adenosine triphosphate (ATP) or coenzyme A are imported via specific receptors. Fatty acids utilized in peroxisomal ß-oxidation are imported by membrane bound ATP-binding cassette (ABC) transporters, commonly reported as ABCD proteins (Wanders et al. 2007). One of these fatty acid transporter proteins, $\mathrm{ABCD} 3$, also known as peroxisomal membrane protein 70 (PMP70) is used as a structurally marker of peroxisomes (Rivera-Zavala et al. 2011). Correct functioning of the transport systems assures sufficient supply of substrates and cofactors, essential for peroxisomal metabolic tasks, particularly the two fundamental, fatty acid oxidation (FAO) and $\mathrm{H}_{2} \mathrm{O}_{2}$ metabolism. 


\subsection{Peroxisomal fatty acid oxidation}

Kidney cortex and specifically proximal tubules are heavily dependent on fatty acids as the major energy source (Le Hir and Dubach 1982). In prokaryotic organisms, ß-oxidation of fatty acid is exclusive to peroxisomes, while in eukaryotes, peroxisomes tightly cooperate with mitochondria and form a strategic partnership in cellular energy metabolism (Bonekamp et al. 2009). Whereas mitochondria preferentially oxidize short and medium chain fatty acids, peroxisomes ß-oxidizes almost exclusively the very long chain fatty acids (VLCFA), with more than 22 carbon atoms (Mannaerts and Van Veldhoven 1993). Import of fatty acids into peroxisomes is executed by three different $\mathrm{ABCD}$ proteins $(\mathrm{ABCD} 1, \mathrm{ABCD} 2, \mathrm{ABCD} 3)$, as mentioned above. Peroxisomes enzymatically cleave and shorten the long chain of VLCFA, which are afterwards transported to mitochondria for the completion of oxidation to acetyl-coA (Reddy and Hashimoto 2001). Peroxisomes lack respiratory chain enzymes, and therefore, peroxisomal ß-oxidation is not directly coupled to oxidative phosphorylation and generation of ATP. Hence, most of the produced energy is released as heat. This exothermic nature of peroxisomal oxidation contributes to heat generation in specialized tissues, like brown fat, abundant in hibernating mammals (and also newborns) that do not shiver. The oxidation of fatty acids in peroxisomes is unique due to generation of $\mathrm{H}_{2} \mathrm{O}_{2}$, a byproduct of peroxisomal oxidative reactions. Irrespective of the close interconnection between peroxisomes and mitochondria, enzymatic apparatus for the ß-oxidation differs between these organelles. The first step in peroxisomal ß-oxidation is catalyzed by ratelimiting acyl-CoA oxidase (ACOX), followed by reactions catalyzed by trifunctional protein and 3-ketoacyl-CoA thiolase (Hashimoto 1996; Hunt and Alexson 2008). Dysfunction of any of these enzymes, especially of the rate-limiting ACOX leads to tissue accumulation of 
unmetabolized fatty acids, with potential harmful effects for the cell, as discussed later. Besides the $ß$-oxidation of fatty acids, peroxisomes perform $\alpha$-oxidation of branched fatty acids, such as phytanic acid. Peroxisomes can $\alpha$ - and $\beta$-oxidize a broad range of natural and xenobiotic compounds containing fatty acyl chain, one of the most important tasks in cellular lipid metabolism. In addition, peroxisomes have a key role in biosynthesis of ether phospholipids (plasmalogens) that are highly abundant in nervous system. Depending on cell type and environment, peroxisomes can perform a broad range of metabolic reactions. A short overview of peroxisomal metabolic tasks is presented in Table 1 (adapted from (Smith and Aitchison 2013)). The participation of peroxisomes in metabolism of arachidonic acid and signaling messengers indicates their involvement in cellular signaling and apoptotic pathways (Masters 1996). One of the principal factors involved in activation and proliferation of peroxisomes is peroxisome proliferator-activated receptor alpha (PPAR $\alpha)$. This nuclear transcription factor is stimulated by physiological ligands generated in arachidonic cascade, like leukotrienes, and by treatment with hypolipidemic drugs fibrates. Upon stimulation, PPAR $\alpha$ translocates to nucleus and binds to a particular DNA sequence called PPAR response element (PPRE), located in the promoter region of fatty oxidation enzyme genes (Schrader et al. 2012). The support for role of PPAR $\alpha$ comes also from PPAR $\alpha$ knock-out mice that have basically a normal number of peroxisomes, however an abolished proliferation after stimulation with PPAR $\alpha$ ligands (Lee et al. 1995). In recent years, several PPAR $\alpha$ independent activators of peroxisomal proliferation, such as peroxisome proliferator-activated receptor-gamma coactivator (PGC-1 $\alpha)$ or sirtuin 1 have been reported in the literature (Bagattin et al. 2010; Hasegawa et al. 2010). The later one, sirtuin 1 seems to offer 
protection in tissue injury through positive effects on peroxisomal reactive oxygen species (ROS) metabolism, the second most important task performed by peroxisomes.

\subsection{Peroxisomal metabolism of reactive oxygen species}

Peroxisomal oxidases generate a huge amount of $\mathrm{H}_{2} \mathrm{O}_{2}$ and other ROS, byproducts of their oxidative reactions. This requires a reliable protective scavenger system, exemplified by the highest concentration of catalase in peroxisomes. This dual role of peroxisomes, generation and decomposition of $\mathrm{H}_{2} \mathrm{O}_{2}$, predestinates them for a decisive role in the delicately regulated redox homeostasis of the cell. There is increasing evidence on the close relationship between intraperoxisomal and cellular redox homeostasis. Malfunction of peroxisomal antioxidative mechanisms may lead to leakage of $\mathrm{H}_{2} \mathrm{O}_{2}$ through the damaged membrane in cytoplasm with harmful consequences for other subcellular structures. Inhibition of peroxisomal catalase alters mitochondrial membrane potential and significantly increases the production of ROS in mitochondria (Ivashchenko et al. 2011). Progeric effects of dysfunctional catalase has been demonstrated by Koepke and colleagues (Koepke et al. 2007). Restoration of intraperoxisomal catalase in aging cells led to decrease of cellular senescence, redox equilibrium, improvement of mitochondrial function. On the other side, cellular aging has been associated with peroxisome senescence, decreased import capacity for catalase and subsequently increased ROS and perpetuation of cellular aging. It is important to mention that low levels of ROS may actually function as mediators in cell signaling. The relevance of signaling properties of these low hormetic levels of reactive oxygen species has been described in the stress-response of mitochondria (Balaban et al. 2005). Hydrogen peroxide has been implicated in activation of 
autophagy, a process with critical importance in cellular surveillance, as discussed later in the text (Scherz-Shouval et al. 2007). Thus, at low concentrations, $\mathrm{H}_{2} \mathrm{O}_{2}$ can indeed stimulate anti-aging pathways (activation of cell surveillance and removal of aged organelles), and at higher concentrations exacerbates organellar and cellular aging. Coming back to peroxisomal FAO and ROS metabolism, and recalling the close link with mitochondrial metabolism, it becomes evident that peroxisome-mitochondria partnership must have a fundamental role under physiological as well as under pathological conditions in cellular microenvironment. The rigid view of mitochondria, as the exclusive generator of cellular ROS has been challenged by new discoveries coming from peroxisomal studies. Further support for their communication and cooperation brings the evidence that both organelles share components of their division machinery (Schrader et al. 2012). Taking together, peroxisomal disturbances may result in significant, under certain circumstances fatal consequences in individual cells, organs or even the whole organism, as demonstrated in primary peroxisomal disorders.

\subsection{Peroxisomal disorders}

Hereditary defects of peroxisomes cause devastating human diseases. In general, inborn peroxisomal disorders can be categorized in (1) peroxisomal biogenesis disorders and (2) peroxisomal disorders caused by deficiency of peroxisomal enzymes or transporters. The common characteristic of peroxisomal diseases is the accumulation of unmetabolized VLCFA, branched chain fatty acids, normally processed in peroxisomes. (1) Zellweger syndrome (also called cerebrohepatorenal syndrome) is the prototypal peroxisomal biogenesis disease, caused by mutations in Pex genes that encode specific peroxisomal proteins - peroxins. Depending on the 
mutation, peroxisomes may be completely missing or present as "ghost" empty peroxisomes with severely compromised import (due to defective peroxins of the import apparatus). Patients with Zellweger syndrome show craniofacial dysmorphism, severe neurological symptoms, hepatomegaly and renal dysfunction. Currently, there is no effective treatment of this syndrome, and most Zellweger infants usually do not survive beyond one year of age. (2) Peroxisomal disorders caused by single enzyme or transporter deficiencies result in symptomatic diseases caused by accumulation of the corresponding metabolite (such as phytanic acid in Refsum disease). In peroxisomal enzyme/transporter disorders, patients usually have structurally intact organelles, though, these are dysfunctional. A recent review by Braverman provides more detailed and updated information on peroxisomal disorders (Braverman et al. 2013).

There is increasing evidence, in contrast to severe hereditary peroxisomal disorders, that temporary/minor perturbations of peroxisomal functions may be involved in the molecular pathogenesis of more common human diseases. Similar cellular metabolic abnormalities as described in primary peroxisomal disorders have been for example observed in Parkinson's disease (Yakunin et al. 2010). Peroxisomal participation has also been advocated in the molecular pathogenesis of ß-amyloid-related neurodegeneration in Alzheimer's disease (Inestrosa et al. 2013). New data on the role of peroxisomes in human aging and in the pathogenesis of agerelated diseases, such as type 2 diabetes emphasize the potential of peroxisome-directed therapeutic approaches (Fransen et al. 2013). The emerging evidence that human immunodeficiency virus, influenza or rotaviruses are exploiting peroxisomes for their replicate purposes, open new fields in the investigations of the cellular response against viral infections (Odendall and Kagan 2013). 
To better understand the underlying defects the peroxisomal disorders, or per se functioning of these organelles in health and disease, appropriate experimental models are required. The majority of the data on peroxisomes comes from studies performed in prokaryotic organisms, mostly from peroxisomal mutants generated in different yeasts. Unfortunately, no naturally occurring animal model of peroxisomal dysfunction is known so far. The first animal models were created in 1997 by Baes et al. for Pex 5 and Faust et al. for Pex7 in laboratory mice. Currently, mouse models with deficiency of Pex5, Pex7, Pex11 and Pex13 are available. The common problems observed in these animals is tissue accumulation of VLCFA and extensive neurodevelopmental disturbances. Due to high perinatal and postnatal mortality of peroxin knock-out models, these are not appropriate for exploration of peroxisomal-related pathogenesis based on temporary/minor alterations of peroxisomal function potentially implicated in the pathogenesis of more common diseases. One of the opportunities to overcome these problems is the implementation of conditional knock-out models, whereby peroxisomes are removed in specific tissues and/or at a specific stage. For detailed information about these models and their impact on cellular and organ functions, please refer to recent reviews (Baes and Van Veldhoven 2012; Van Veldhoven and Baes 2013). The natural lack of models of specific peroxisomal defects also implicates rather subtle and/or temporary alterations of peroxisomes in the pathogenesis of common disorders. These minor changes of peroxisomes may result from direct or also indirect impairment of their functions, for example by compromised recycling due to defect autophagy. 


\subsection{Peroxisomal homeostasis and autophagy}

Cells regulate the number of the organelles through continual interplay between biogenesis and degradation. Constant turnover of peroxisomes is essential for preservation of their functional competence. Experimental studies in mouse fibroblasts demonstrated that peroxisomes have a half-life of circa 48 hours under basal conditions and are mainly degraded by autophagy (Huybrechts et al. 2009). Old human skin fibroblasts accumulate functionally impaired peroxisomes, and the consequently increased oxidative stress additionally accelerate cellular aging (Legakis et al. 2002). Autophagy is a highly conserved lysosome-dependent pathway maintaining the quality of intracellular constituents through continual elimination of redundant or damaged proteins or even whole organelles (Mizushima and Komatsu 2011). Molecular basis of autophagy-mediated degradation of peroxisomes, so-called pexophagy has been extensively studied in yeast and reviewed in (Till et al. 2012). Contrary to yeast, exact details about pexophagy in mammalian cells are not completely understood. Yokota et al. described three independent clearance systems of peroxisomes: (1) Lon protease system, (2) 15-lipooxygenasemediated membrane lysis and (3) lysosomal autophagy (Yokota and Dariush Fahimi 2009). Briefly, Lon protease is an ATP-dependent enzyme that catalyzes proteolytic digestion of peroxisomal proteins, and accounts for removal of about $20 \%$ of peroxisomes. The activation of cytosolic 15-lipooxygenase can induce peroxisome membrane disruption, eliminates though only a small fractions of peroxisomes (Yokota et al. 2001). The most important pathway responsible for removal of the largest portion of peroxisomes is lysosomal autophagy. Whereas in yeast, induction of autophagy is mainly a metabolic adaptation to altered environmental/culture conditions (in order to maintain nutritive balance under starvation), in mammalian cells, 
autophagy functions primarily in quality control and homeostasis surveillance through elimination of aged/damaged cellular components. Three main types of autophagy have been characterized: macroautophagy, microautophagy and chaperone-mediated autophagy (Todde et al. 2009). Macroautophagy is the main type of organellar autophagy in mammalian cells. For this reason, by using the term "autophagy", I refer to macroautophagy in the further text. Autophagy begins with formation of a double membrane structure around cytoplasmic component(s), socalled autophagosome. Autophagosome with engulfed proteins or organelles travels through the cytoplasm and fuses with lysosome. Selective autophagy of peroxisomes requires the presence of specific cargo receptors (Till et al. 2012). From the ensemble of factors involved in autophagy, the p62/SQSTM1 protein has been directly associated with mammalian pexophagy. Autophagy recognizes monoubiquitinated proteins. This is supported by experimental data demonstrating that monoubiquitination of peroxisomal membrane proteins induced pexophagy of such "labelled" peroxisomes (Kim et al. 2008). The p62/SQSTM1 has an ubiquitin-binding domain (UBD) and a LC3-interracting region (LIR) (Behrends and Fulda 2012). Upon induction of autophagy, cytosolic LC3 (microtubule-associated protein light chain 3) is recruited to autophagosomal membrane. The p62/SQSTM1 binds with UBD the ubiquitinated proteins on peroxisomal membrane and via LIR the LC3 on autophagosomal membrane, and docks so peroxisomes to autophagosomes. The fusion of autophagosome with lysosome creates an autolysosome (autophagy lysosome), the place of the final degradation and recycling of cytosolic components (Figure 2). Because p62/SQSTM1 is degraded by autophagy at the same time as the ubiquitinated cargo, it has been used as a marker of autophagy flux. In a simple view, the inhibition of autophagy leads to accumulation of p62/SQSTM1 and vice-versa. The 


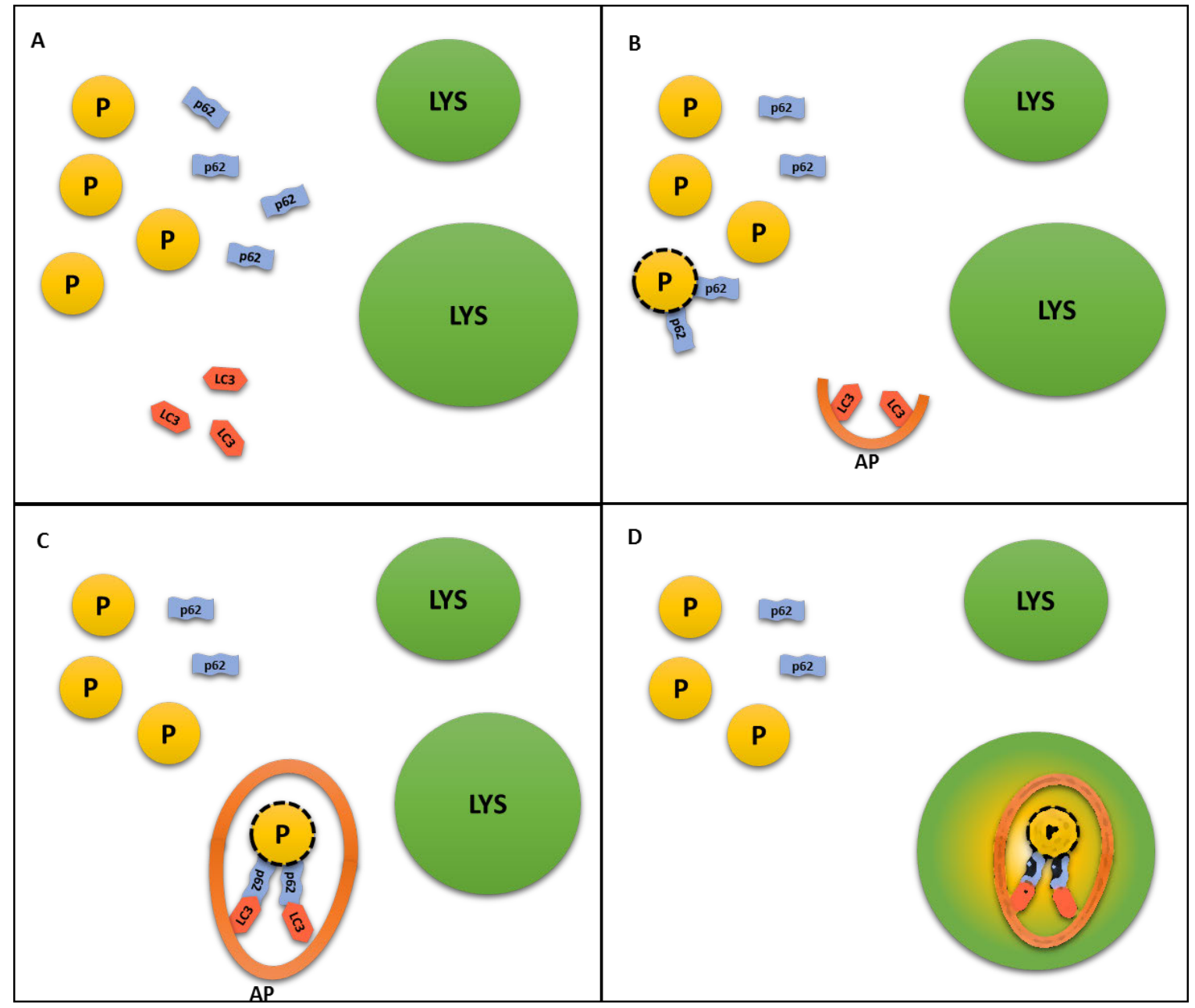

Figure 2. Autophagy of peroxisomes - pexophagy. (A-B) Upon activation of pexophagy, the p62 protein binds to peroxisomal membrane via its ubiquitin-binding domain. LC3 protein is recruited from cytosol to newly formed and expanding autophagosomal membrane (AP). (C) Then, p62 attached to peroxisome binds to LC3 on autophagosome via its LC3-interracting region (D). Finally, the closed autophagosome with engulfed peroxisome $(\mathrm{P})$ travels to and fuses with lysosome (LYS).

accumulation of peroxisomes in p62/SQSTM1 knock-out models corroborates the role of this protein in pexophagy. An important prerequisite for accurate functioning of autophagy is the preserved motility of peroxisomes. Pex14, a membrane peroxin functioning in protein import can interact with tubulin, which enables peroxisomes to move across the cell. This is supported by 
experimental data demonstrating that Pex14-deficient peroxisomes lose their ability to move along microtubules (Bharti et al. 2011).

\subsection{Peroxisomes and kidney}

Renal tubular cells and hepatocytes have the highest density of peroxisomes. Renal tubular cells rely on FAO and disturbances of this indispensable metabolic pathway aggravate renal injury. Dysfunctional and inhibited beta-oxidation in mitochondria and peroxisomes results in increased levels of cytotoxic free fatty acids (backward failure) and decreased generation of energy (forward failure) in renal parenchyma. Much of the early data on peroxisomes in renal pathology originated in studies with ischemia-reperfusion models of acute kidney injury (AKI).

Ruidera et al. examined catabolism of free fatty acids and demonstrated that 60 minutes of renal ischemia reduced the oxidation of fatty acids by $57-69 \%$, depending on the substrate (Ruidera et al. 1988). Gulati et al. showed that $90 \mathrm{~min}$ of renal ischemia caused significant loss of peroxisomal matrix proteins, reflected by a significant decrease in catalase $(35 \%)$ and betaoxidation activity (43\%) for C24 lignoceric acid. The authors further demonstrated that kidney is capable to restore beta-oxidation and catalase activity after ischemia not exceeding 60 minutes. Longer periods of ischemia were associated with degradation and irreversible damage of peroxisomes (Gulati et al. 1992). The same group reported that major loss of the enzymatic activities during ischemia was due to enzyme inactivation, whereas during reperfusion, proteolytic degradation of the enzymes followed. Among peroxisomal FAO enzymes, the rate-limiting ACOX is more sensitive to ischemic cellular stress, and hence the major contributor to loss of beta-oxidation in ischemia-reperfusion injury (Gulati et al. 1993). High tissue levels of free fatty 
acids are cytotoxic, causing destabilization of mitochondrial potential, inhibition of proximal tubule $\mathrm{Na}^{+} \mathrm{K}^{+}$-ATPase and stimulation of inflammatory cytokines production (Portilla 1999; Feldkamp et al. 2006). The negative consequences of inhibited FAO in AKI initiated studies that analyzed possible protective effects of FAO stimulation. Peroxisome proliferator-activated receptors belongs to nuclear receptors family and function as ligand-activated transcription factors. Clofibrate, one of the early cholesterol-lowering drugs has been shown to stimulate proliferation of peroxisomes in rat. The receptor mediating this effect was later identified and named PPAR $\alpha$ (Hess et al. 1965; Issemann and Green 1990). Peroxisome proliferator-activated receptor alpha (PPAR $\alpha)$ is the principal stimulator of peroxisomes (see section 1.2 Peroxisomal fatty acid oxidation). Renal PPAR $\alpha$ resides predominantly in the proximal tubule, corresponding with the highest density of peroxisomes in this part of the nephron (Huang et al. 1995). Cisplatin, a recognized nephrotoxin, reduces FAO in proximal tubule by inhibition of PPAR $\alpha$ binding to DNA, reflected by decreased expression of mitochondrial carnitine palmitoyl transferase (CPT) and peroxisomal ACOX (Portilla et al. 2002). Co-administration of PPAR $\alpha$ ligand in cisplatin-treated mice ameliorated renal injury through activation of mitochondrial medium chain acyl-CoA dehydrogenase (MCAD). This effect was eliminated in cisplatin-treated PPAR $\alpha$ knock-out mice (Li et al. 2004). Transgenic mice overexpressing PPAR $\alpha$ in proximal tubules showed improved kidney function and morphology during cisplatin or ischemia-induced renal injury, mainly through improvement of fatty-acid metabolism and mitochondrial oxidative phosphorylation, (Li et al. 2009). PPAR $\alpha$ transgenic mice also showed decreased expression of inflammatory and pro-fibrotic cytokines in unilateral ureteral obstruction, an experimental model of renal fibrosis (Li et al. 2013). 
Sirtuin-1 is a NAD-dependent deacetylase regulating numerous cellular functions, and has been associated with longevity in mammals. In the kidney, SIRT1 inhibits cell apoptosis, reduces inflammation, improves lipid metabolism and autophagy, to name a few (Borradaile and Pickering 2009). Transgenic mice with overexpression of sirtuin 1 in proximal tubules could maintain peroxisome number and functionality of catalase, showed by decreased levels of ROS, and diminished apoptosis of renal tubular cells during cisplatin-induced AKI (Hasegawa et al. 2010). Protective effects of sirtuin 1 in acute and chronic renal injury has been reported recently also by our group (Vasko et al. 2014).

Most of the previous studies focused on mitochondria and neglected peroxisomes. Studies that examine regulation of mammalian peroxisomes in the pathogenesis of kidney diseases are very scarce, and therefore, solid data on their role in renal injury are still almost non-existent. 


\section{Peroxisomes and pexophagy in LPS-induced acute kidney injury}

The role of peroxisomes and pexophagy in the pathogenesis of acute kidney injury (AKI) has not been studies yet. The aim of our study was to examine, what is the default response of peroxisomes to endotoxic stress, and how does a preexisting dysfunction of lysosomal autophagy/pexophagy impact the regulation of peroxisomes. To accomplish this, we studied the impact of LPS on peroxisomes in vivo, in mice with impaired lysosomes, and in vitro in cells with pharmacological inhibition of lysosomal function.

LPS-mediated injury represents a perfect model of cellular stress challenging metabolic pathways and accompanied by substantially increased levels of ROS, two tasks with close connection to peroxisomes.

Induction of autophagy helps to maintain cellular homeostasis during stress. It has been argued that enhanced autophagy of damaged mitochondria (mitophagy), and consequently decreased release of caspases-activating cytochrome $\mathrm{C}$ from injured mitochondria is responsible for the improved viability during cellular stress (Ravikumar et al. 2006). Considering the tight link between mitochondrial and peroxisomal intraorganellar homeostasis, here, we offer an additional explanation, namely that autophagic degradation of peroxisomes (pexophagy) also contributes to cell survival during stress.

\subsection{Acute kidney injury in mice with impaired pexophagy}

All experimental animals used in our study were treated in accordance with the NIH Guide for the Care and Use of Laboratory Animals and the study protocol was approved by the Institutional 
Animal Care and Use Committee. The experiments were performed using 12 to 14-week-old C57BL/6 (wt, wild-type mice) and C57BL/6J-Lyst ${ }^{\text {bg-J }}$ mice (lys, lysosomal mice). Lys-mice have the same genetic background as wild-type mice. They carry a mutation in lysosomal trafficking regulator protein, and represent a model of impaired lysosomal function and autophagy. AKI was induced by injecting the mice with $5 \mathrm{mg} / \mathrm{kg}$ dose of LPS. One could argue, why was the dose of LPS used relatively low and why did we conduct the study in adult, rather than younger mice. For that reason, before proceeding further, I would like to briefly discuss several important issues in animal models of septic AKI.

Firstly, susceptibility of mice to acute renal injury in sepsis appears to be significantly influenced by genetic factors, in case of wild-type animals mainly by specific strain. Whereas for example CD-1 mice are prone to develop septic kidney injury at a younger age, the C57BL/6 mice, also used in our study, develop endotoxin-induced renal injury consistently at an advanced age. That was the reason why we have decided to use adult mice, and not the 6-8 week-old mice usually reported in literature. In our previous studies with C57BL/6 mice strain, we could observe a consistent renal sequel to LPS treatment in adult mice compared to variable response in younger counterparts. This is in agreement with data reported by other groups. Secondly, the dose of LPS used in our study, $5 \mathrm{mg} / \mathrm{kg}$, is a non-lethal dose reliably inducing kidney injury, not an organ failure. In our other studies with higher doses of LPS $(12-18 \mathrm{mg} / \mathrm{kg})$, the treated animals exhibited high mortality and the survivors showed significant anomalies, involving anorexia, locomotor inhibition, hypotension and organ dysfunction; all consistent with clinical picture of septic shock. This is one explanation for the shorter duration (12 hours) of the studies using higher doses of LPS. 
In addition, we measured vital parameters in our mice, and did not observe differences between lys- and wt-animals under normal conditions, and after treatment with LPS. Except of the slightly increased heart rate, there were no significant changes in mice treated with LPS compared to untreated animals. Hence, LPS at the dose used did not result in systemic hypotension (shock), which would have aggravated kidney injury due to organ hypoperfusion.

Under basal conditions, wt- and lys-mice did not differ in terms of serum creatinine levels, although lys-mice showed a mild proteinuria. After introduction of endotoxic stress by a single injection of a non-lethal dose of LPS, lys-mice developed significantly higher proteinuria and increased serum creatinine levels compared to wt-mice (see Vasko et al., Fig. 1, p. 213). Histopathological examination of the kidneys from animals sacrificed 24 hours after LPS treatment demonstrated different pattern of injury in wt-mice and lys-mice. Whereas in wt-mice, we could observe predominantly swelling of epithelial cells with focal desquamation of the brush borders, the injury areas in lys-mice demonstrated marked swelling of the epithelial cells, desquamation and focal necrosis. Any of the animals demonstrated severe kidney damage with extensive necrosis, which was consistent with our intention to study acute organ injury and not an acute organ failure. In agreement with this objective, LPS-treated lys-mice developed a smaller increase of s-creatinine $(0.5 \mathrm{mg} / \mathrm{dl})$ compared to other models of AKI used in our lab (e.g., in acute folic acid nephropathy $-1.0 \mathrm{mg} / \mathrm{dl}$, in ischemia-reperfusion model $-2.0 \mathrm{mg} / \mathrm{dl}$ ), which also demonstrated more extensive renal damage.

Because of different severity of the kidney injury in wt- and lys-mice, we were interested, if there are any differences in cytokine milieu between these animals. We obtained sera from mice under 
normal conditions and after LPS injection, and examined the concentrations of pro-inflammatory cytokines and chemokines using Luminex analyzer.

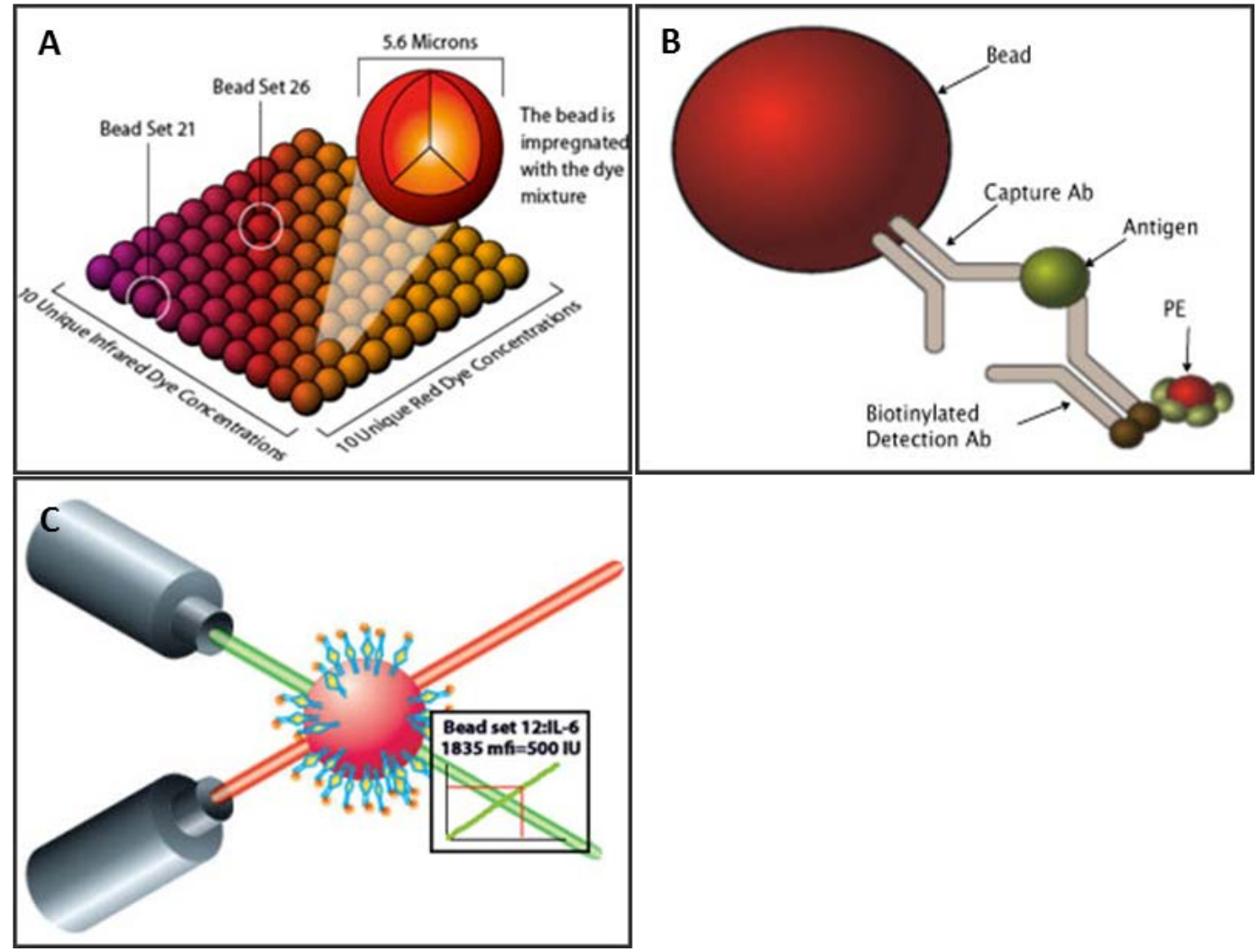

Figure 3. Principle of Luminex Multiplexing Assay. Explanation see in text. (Reproduced with permission of the copyright owner, Roswell Park Cancer Institute, Buffalo, New York, USA)

Luminex is a bead based multiplexing technique, based on the xMAP (any Multiple Analyte Profiling) technology which recognizes color-coded microspheres generated by combinations of 10 shades of 2 dyes (Figure 3A), producing so a 100 bead sets. Each bead can be coated with a specific antibody and binding of the analyte can then be quantified using reporter antibodies (Figure 3B). Luminex technology allows discrimination of up to 100 unique assays within a single sample (theoretically 100 antigens in one well of the 96-well plate). The Luminex 
instrument has 2 lasers in which one is used to excite and detect a specific bead and the other to detect the fluorochrome (phycoerythrin) bound to a reporter antibody (Figure 3C).

Lys-mice showed increased serum levels of G-CSF/GM-CSF under basal conditions, and significantly higher inflammatory burst with increase of IL-6, IL-8, TNF-alpha and MCP-1 after LPS injection compared to wt-mice. Similarly as in humans, we have only limited data to be able to establish reference range for serum concentrations of cytokines (perhaps except IL-1, IL-6). The differences in cytokine/chemokine levels are therefore presented relatively to controls (see Vasko et al., Fig. 2, p. 214),

Conclusively, our data demonstrate aggravated functional, morphologic and inflammatory responses in lys-mice, but do not reveal the site(s) governing these responses.

\subsection{Endothelium, sepsis and cytokine storm}

Renal capillary endothelial cells form a dense three-dimensional system surrounding all structures in the kidney. Due to the enormous density of around 5,000 cells per square millimeter and their strategic position between circulating blood cells on one side, and tubular cells on the other side, endothelium has a key commanding role in the crosstalk within the renal microenvironment. Transgenic models (Andonegui et al. 2009) and recent evidence from pulmonary influenza infection (Teijaro et al. 2011) provide new insights on the role of endothelial cells as crucial sentinels in detection and clearance of infectious agents and their products. LPS activation of endothelial cells with resulting cytokine surge has a profound impact on pericapillary environment with the residing cells. Capillary dysfunction is an early event in sepsis and has a 
significant impact on the outcome of AKI (Wu et al. 2007). The previously held view that cytokines are produced mainly by epithelial cells is lately shifting towards the realization that endothelial cells are the principal orchestrators of cytokine responses in sepsis (Harding and Kubes 2012; Steinberg et al. 2012). Previous studies demonstrated that inflammatory signaling through TNF receptor 1 is crucial for AKI (Cunningham et al. 2002; Cunningham et al. 2004). The expression of TNF receptor 1 in the kidney is mainly endothelial and not tubular (Eadon et al. 2012). Even an ascending urinary infection produces alterations in peritubular capillaries as early as 3 hours after bacterial adhesion to surface of renal tubular cells (Melican et al. 2008).

We isolated primary endothelial cells from wt- and lys-mice, and analyzed their secretomes for cytokine concentrations in parallel with the sera obtained from these animals. Cytokine profiling of these cells revealed similar patterns as seen in vivo in mice, which is consistent with the proposed preeminent role of the endothelium in LPS-induced "cytokine storm".

Our decision to use endothelial cells was not based merely on the recent data discussed above. We wanted to make evident how important is the close relationship between these two neighboring systems, and did not intend just simply adapt the behavior of endothelial to tubular cells. We do believe that new understandings will soften the classical epithelial-centered dogma, not only in preparation of studies but also in interpreting and discussion of the results.

\subsection{Renal peroxisomes in endotoxic stress}

To study the behavior of renal peroxisomes, we examined the expression of selected specialized peroxisomal proteins, peroxins (see Introduction) and catalase in kidney lysates obtained from wt- 
and lys-mice using immunoblot (see Vasko et al., Fig. 3, p. 215). PMP70 is a 70-kDa peroxisomal membrane integral protein, a major components of the membrane, and is used as a structural marker of peroxisomes. PMP70, also known as ABCD3 protein participates in fatty acid import for peroxisomal FAO. Pex14 is a peroxisomal membrane-associated protein (not entirely integrated and immobilized as PMP70), a key component of the peroxisomal import machinery. Pex14 binds and facilitates translocation of PTS1 and PTS2 labeled proteincomplexes across the peroxisomal membrane. Pex $11 \mathrm{~b}$ is a regulatory peroxin involved in control of peroxisomal proliferation and division. Under basal conditions, lys-mice showed higher levels of PMP70 and Pex14 than wt-mice, a consequence of decreased clearance by dysfunctional lysosomal autophagy/pexophagy. This is also supported by accumulation of p62/SQSTM1 in these animals, a marker protein of the autophagic flux. Treatment with LPS in wt-mice resulted in robust upregulation of PMP70, Pex14 and Pex11 expression. In lys-mice, LPS caused only minor changes of PMP70 and Pex11 levels, and decreased the expression of Pex14. Interestingly, compared with wt-mice, lys-mice showed increased protein levels of catalase under basal condition. LPS treatment resulted only in minor changes of catalase in lys-mice, and in significant decrease of catalase in wt-mice.

Taken together, these data demonstrate that treatment with LPS stimulates proliferation of peroxisomes in wt-mice and produces only a blunted response in lys-mice. The abolished expression of Pex11 in lys-mice supports the assumption of impaired proliferation of peroxisomes. 


\subsection{Endothelial peroxisomes in endotoxic stress}

Our assumption of impaired peroxisomal proliferation/division in lys-mice is also supported by distinct morphological features of peroxisomes in endothelial cells isolated from these animals. Lyst-endothelial cells exhibited higher prevalence of enlarged and elongated forms, suggestive of a defect in proliferation and fission (see Vasko et al., Fig. 4A, p. 217). Primary murine endothelial cells were explant cultures isolated from aortas of experimental animals, and passages 2-3 were used in our experiments. The second more sophisticated approach we have used, was the isolation of primary renal endothelial cells using dynabeads and enzymatic digested kidney tissue. Dynabeads are superparamagnetic spherical particles linked to an antibody that recognizes a specific antigen. Using dynabeads coated with CD31 (PECAM-1) monoclonal antibody, we were able to perform immunomagnetic separation of endothelial cells.

Because of the very slow growing characteristics of primary murine endothelial cells and limited quantity of material available, we decided further to explore the regulation of endothelial peroxisomes using primary human umbilical vein endothelial cells (HUVEC), passages 2 to 5. In our preliminary experiments, we tested the toxicity of different LPS concentrations on HUVEC, and observed a significant decrease of cell viability when exceeding the concentration of $3 \mu \mathrm{g} / \mathrm{ml}$. We used very early HUVEC passages, cultured in a special endothelial growth medium tested for contamination with endotoxin. The effect of LPS on endothelial cells was studying using concentration of $1 \mu \mathrm{g} / \mathrm{ml}$. Lysosomal dysfunction was induced by pretreatment with lysosomotropic drug chloroquine. Primary endothelial cells isolated from lys-mice differ from cells isolated from wt-mice due to their larger volume and course granular appearance. Primary 
HUVEC treated with chloroquine developed similar phenotypic features as lys-endothelial cells isolated from lys-mice (Figure 4).

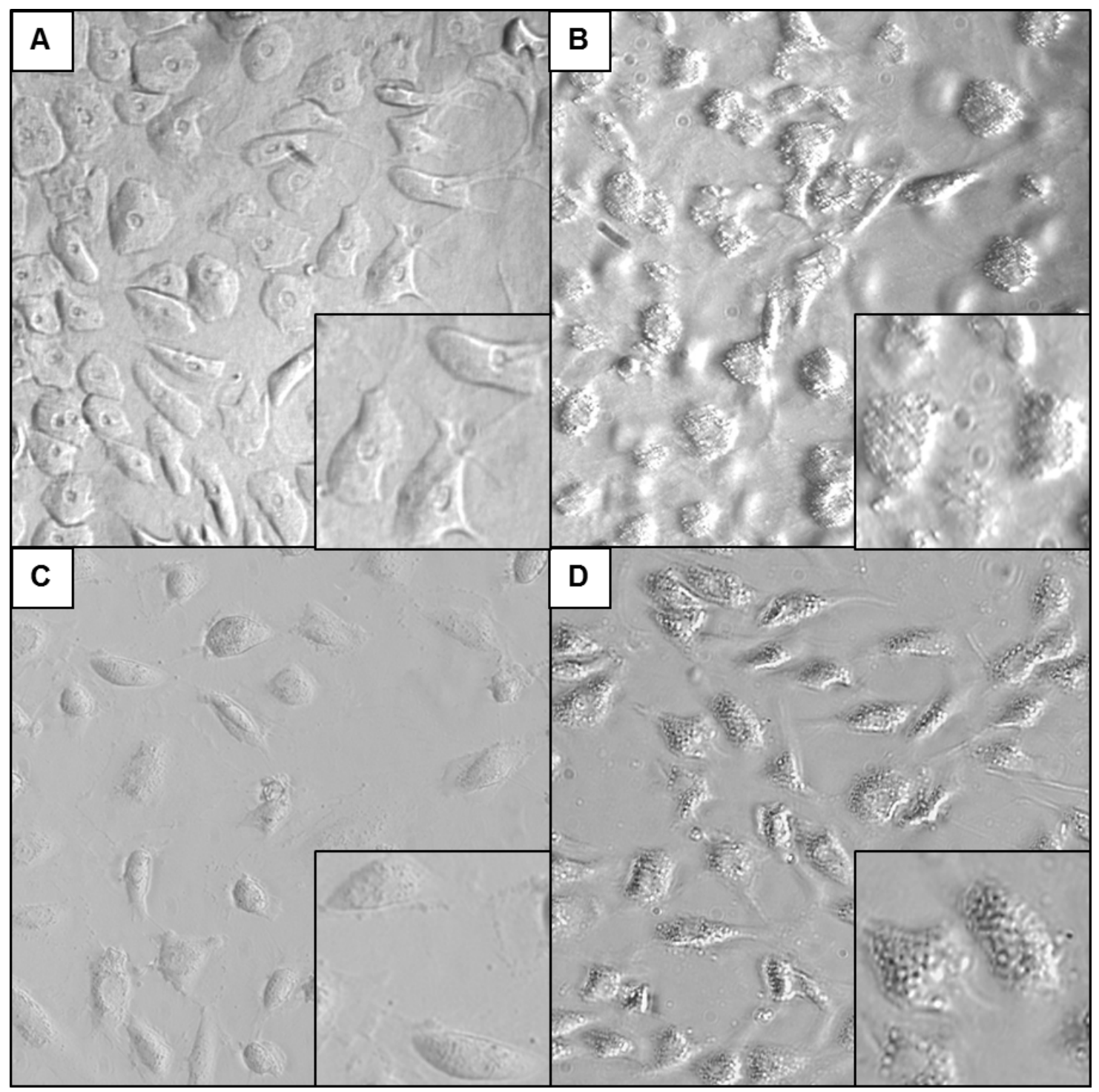

Figure 4. Primary endothelial cells with hereditary (lys-mice) or chloroquine-induced lysosomal dysfunction. (B) Endothelial cells isolated from lys-mice with impaired lysosomal autophagy showed a different morphology with larger cellular volumes and granular appearance when compared to (A) endothelial cells from wild-type mice. (D) Human umbilical vein endothelial cells (HUVEC) with chloroquine-induced lysosomal dysfunction acquired similar phenotypic features as lyst-endothelial cells. (C) HUVEC cultured under normal conditions. Magnification 400x. 
Detailed examination of peroxins in LPS-stimulated HUVEC demonstrated a biphasic expression of PMP70, Pex14 and catalase, with an initial decline followed by a gradual increase of protein expression (see Vasko et al., Fig. 5, p. 218). The impact of LPS on Pex14 was more profound than on PMP70, similar to effects observed in kidney. We verified the expression of PMP70 in a small number of available primary murine endothelial cells (see isolation above) and observed a biphasic pattern as seen in HUVEC, confirming the suitability of HUVEC as a culture model (data not shown). The decrease of p62/SQSTM1 after LPS stimulation indicates an increased autophagic flux and activation of lysosomal pexophagy. (Figure 5) nicely demonstrates decreased density of peroxisomes in the vicinity of lysosomes after LPS exposure. Pre-treatment of HUVEC with chloroquine compromised lysosomal function and autophagy, led to accumulation of p62/SQSTM1 and loss of the biphasic PMP70 and Pex14 expression seen in control cells with competent lysosomes after stimulation with LPS (see Vasko et al., Fig. 6, p. 219). Immunofluorescence studies confirmed the expression changes of PMP70 and Pex14 described above (see Vasko et al., Fig. 7, p. 220). Looking in more detail at the expression of PMP70 and Pex14 in chloroquine pretreated cells after 24 hours, it becomes obvious that the expression of both is slowly declining at this late time-point (see Vasko et al., Fig. 6B+D, p. 219). In parallel, p62/SQSTM1 protein starts to accumulate at the later stages of the experiment (see Vasko et al., Fig. 6E p. 219). These changes indicate worsening of peroxisomal homeostasis at advanced stages of LPS treatment in lysosome-deficient cells.

Lysosomal dysfunction suppressed autophagic degradation of peroxisomes, pexophagy and prevented recycling of peroxisomes during LPS stress. These in vitro findings in endothelial cells 
corroborate similar changes in the whole organ responses to LPS and emphasize the role of autophagy/pexophagy in this process.

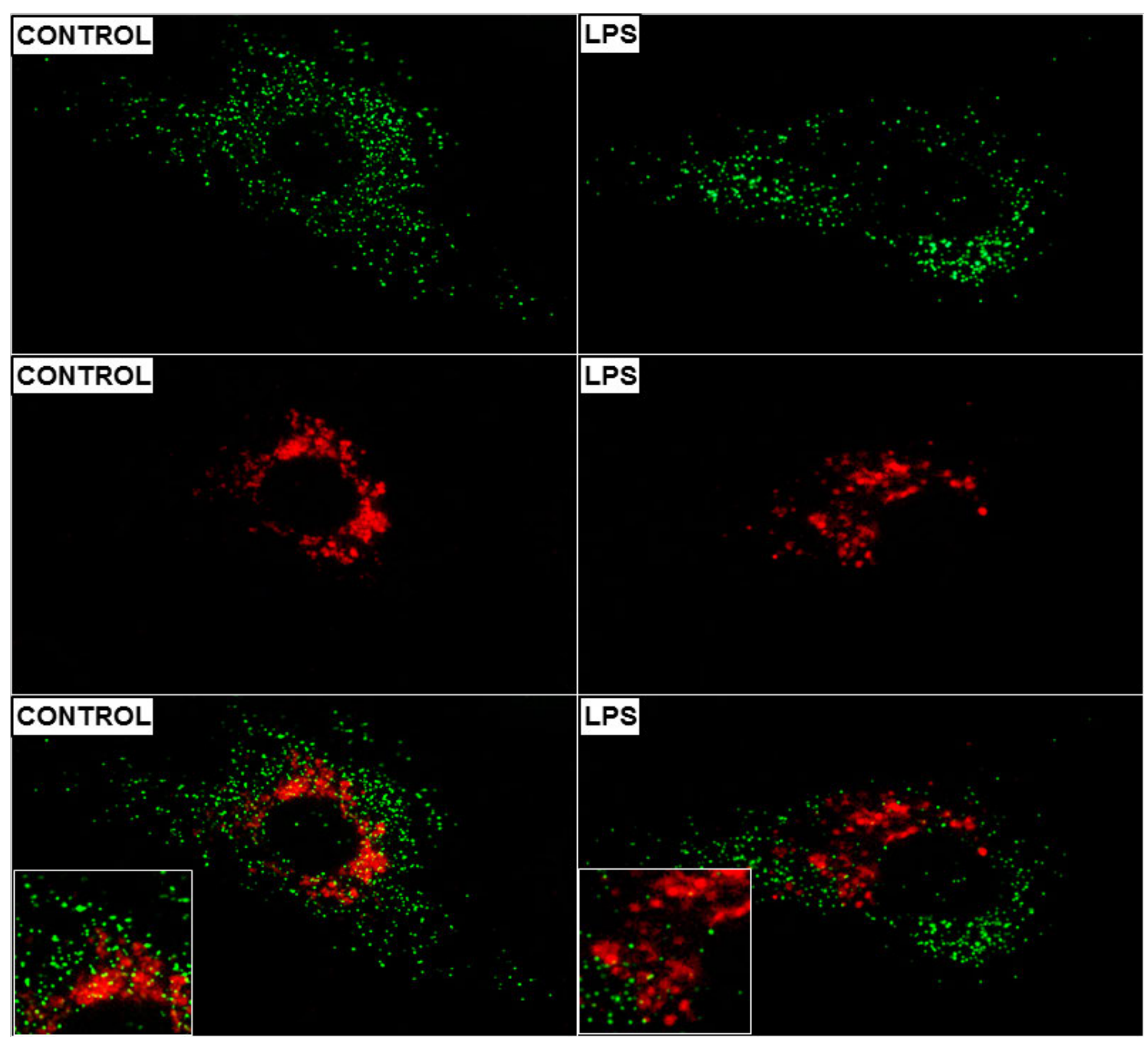

Figure 5. Representative images of human umbilical vein endothelial cells (HUVEC) with green labeled peroxisomes and Lysotracker stained red lysosomes. HUVEC were transfected with green fluorescent protein fused to peroxisomal targeting signal type 1 (PTS1). Cells were treated with 1 $\mu \mathrm{g} / \mathrm{ml}$ LPS for 6 hours (LPS). Untreated cells served as a control (CONTROL). To demonstrate the spatial relationship of peroxisomes and lysosomes, images were taken sequentially through the whole cell volumes and then overlapped. There is evident reduction of peroxisomal density in the areas within close proximity to lysosomes in cells treated with LPS. 


\subsection{Peroxisomal import and LPS}

From the presented data, it is apparent that LPS exhibits more profound effect on Pex14 than PMP70 expression. Pex14 is a key component of the peroxisome protein import machinery and facilitates the import of PTS1 containing proteins (see Introduction). Therefore, we proposed that treatment with LPS would have impact on peroxisomal import. To prove this theory, we transduced primary endothelial cells with PTS1-GFP and examined the effect of LPS on peroxisomal import by colocalization of PTS1-GFP with peroxisomal marker protein PMP70. Transduction was performed using baculovirus-mediated transgene delivery. The vector contains enhanced CMV promoter, C-terminal peroxisomal targeting signal 1 (PTS1) fused to emerald green fluorescent protein. This technique delivers highly reproducible and titratable expression, with high efficiency and minimal cytotoxicity also in early endothelial cell passages, which were almost resistant to our preliminary transfection attempts with electroporation. The colocalization ratio of PMP70 with PTS1-GFP represents the proportion of peroxisomes which imported the PTS1-GFP construct. Under normal conditions, the colocalization between PMP70 and PTS1GFP was $82 \pm 6 \%$. After stimulation with LPS, (in overall reduced peroxisomal population) colocalization decreased to $46 \pm 5 \%$ after 9 hours, and increased again to $63 \pm 4 \%$ after 24 hours, resembling the biphasic expression profile of Pex14 seen in the previous experiments. Interestingly, when examining the proportion of total PTS1-GFP that colocalized with PMP70 (= proportion of the imported PTS1-GFP from the total PTS1-GFP), this increased from 74\% under normal conditions to $89 \%$ after $9 \mathrm{~h}$, and $85 \%$ after $24 \mathrm{~h}$ of LPS treatment (see Vasko et al., Fig. 8 p. 221). In other words, under normal conditions, $82 \%$ of peroxisomes imported in average $74 \%$ of available PTS1-GFP. Nine hours after LPS treatment, merely $46 \%$ of 
peroxisomes imported in average $89 \%$ of available PTS1-GFP. And, 24 hours after LPS treatment, about $63 \%$ of peroxisomes imported in average $85 \%$ of available PTS1-GFP. Hence, after LPS application, a smaller number of residual peroxisomes import PTS1 proteins with relatively higher efficiency. However, in total, the residual (smaller) peroxisomal fraction, irrespective of the increased effort, could not sufficiently substitute for the global import insufficiency (Figure 6).

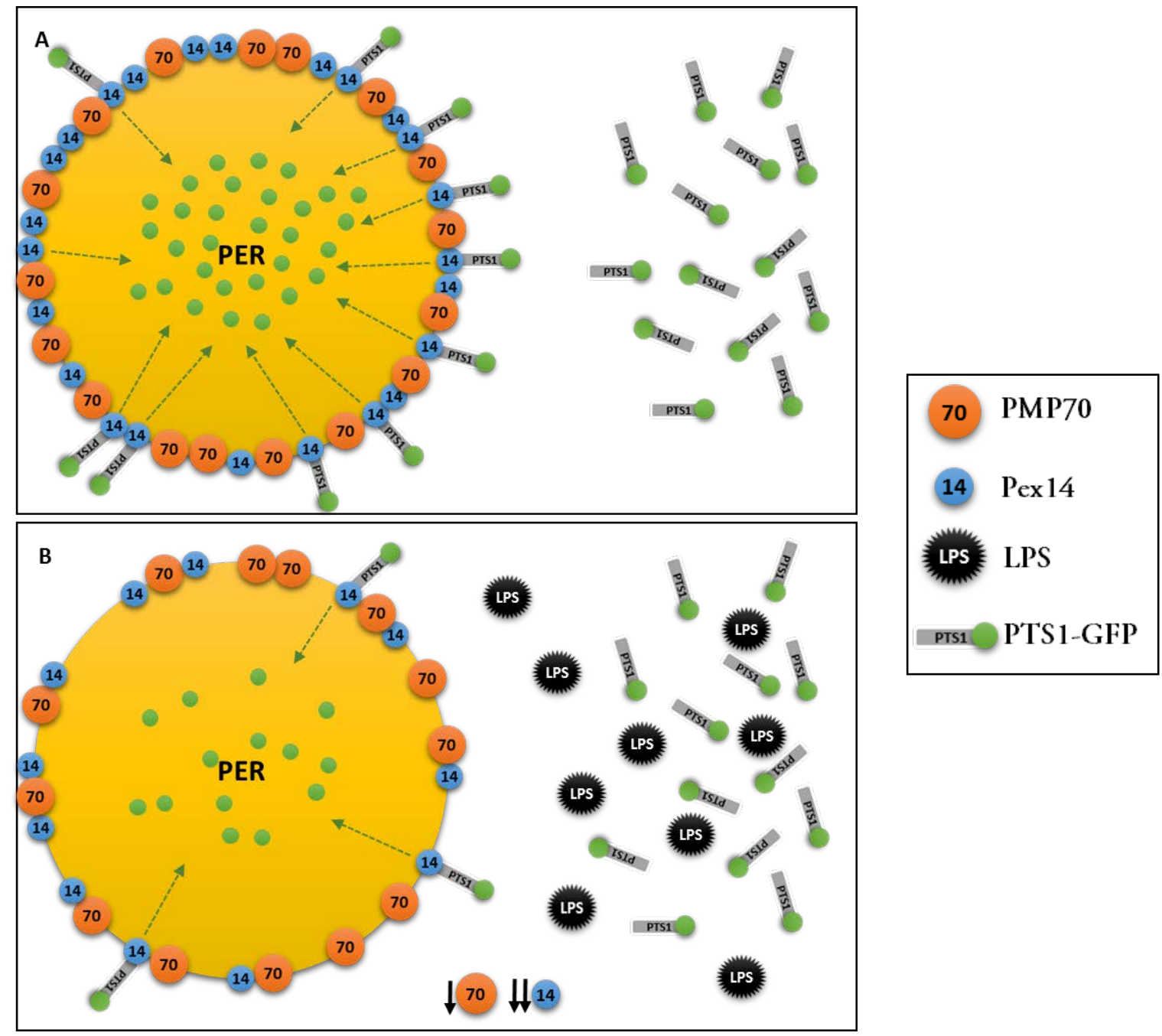

Figure 6. Schematic representation of impaired peroxisomal import after LPS exposure. LPS exhibits more profound effect on Pex14 than PMP70 expression. LPS-induced repression of Pex14 results in decreased import of PTS1-GFP into peroxisomes. 
One of the possible explanations may be that PMP70 is a large membrane-incorporated protein that resists endotoxic stress better than the smaller membrane-associated Pex14. There is also some evidence that Pex14 may play a direct role as a receptor for pexophagy by interacting with p62/SQSTM1 or LC3-II proteins, which could also explain its predominant consumption after LPS (Hara-Kuge and Fujiki 2008). Peroxisomal chief anti-oxidative enzyme catalase has an atypically weaker PTS1 (Purdue and Lazarow 1996). Thus, LPS-induced repression of Pex14 may impair intra-organellar import of proteins, particularly of catalase and aggravate the adverse effects of already decreased catalase protein during LPS exposure (as shown by immunoblot).

\subsection{Lysosomal dysfunction and reactive oxygen species}

Lipopolysaccharide is a potent inductor of cellular ROS. Interestingly, we saw that lys-mice with hereditary impairment of lysosomes and also cells with pharmacological lysosomal dysfunction, both have increased levels of catalase protein under basal conditions. In subsequent studies, we investigated if these are also protective under conditions of increased oxidative stress after LPS. The production of ROS in cells was measured using color change based on oxidative conversion of dichlorodihydrofluorescein diacetate (DCFH-DA, a more general indicator of ROS). To be more specific in assessment of peroxisomal catalase, we also specifically measured the levels of released $\mathrm{H}_{2} \mathrm{O}_{2}$. Both approaches revealed that presence of dysfunctional lysosomes result in higher and protracted levels of $\mathrm{ROS} / \mathrm{H}_{2} \mathrm{O}_{2}$ (see Vasko et al., Fig. 9 p. 222). This is interesting keeping in mind that cells with dysfunctional lysosomes have actually higher levels of catalase protein. For that reason, we next examined explicitly the enzymatic activity of catalase in live cells with or without lysosomal dysfunction. Contrary to lysosome-defective cells that showed 
decreased enzymatic activity of catalase, cells with functional lysosomes had constantly higher catalase activity under basal conditions and after LPS. Pro-inflammatory phenotype with elevated cytokines observed in lys-mice, and increased nitric oxide concentration can interact with haem group and inactivate catalase, without affecting the protein level (Sigfrid et al. 2003).

Peroxisomes are able to handle $\mathrm{H}_{2} \mathrm{O}_{2}$ only up to certain levels. For example mesangial cell exposed to $100 \mu \mathrm{M}$ of $\mathrm{H}_{2} \mathrm{O}_{2}$ upregulated peroxisomal biogenesis, however increasing of the concentration above $250 \mu \mathrm{M}$ had an opposite effect (Hwang et al. 2012). Hydrogen peroxide, particularly when in excess, may leak from peroxisomes, diffuse into lysosomes and form highly reactive hydroxyl radical that further impairs lysosomal function (Terman and Kurz 2013).

\subsection{Metabolic-oxidative imbalance in lysosomal dysfunction}

Summarizing the results of the in vitro experiments, it is evident that the most significant changes in peroxisomal regulation and function occurred relatively short after application of LPS. To verify, if these alterations are also relevant in vivo, we next studied metabolic and oxidative pathways related to peroxisomal functions in kidneys from wt- and lys-mice. In order to examine the dynamics of peroxisomes in vivo, we studied mice treated with LPS for 9 hours (in addition to 0 and 24 hours). Looking at the expression of peroxins, PMP70 and Pex14 demonstrated similar profiles as seen in cultured cells, with initial decline and subsequent increase in wt-mice, which was subverted in lys-mice. Despite the totally increased protein levels, lys-mice demonstrated persistently decreased enzymatic activity of catalase and worse renal oxidative injury. This is buttressed by increased levels of malondialdehyde (lipid peroxidation) detected locally in kidney parenchyma and also in urine from lys-mice after LPS (see Vasko et al., Fig. 
$10 \mathrm{~F}+\mathrm{G}$ p. 223). $\mathrm{FAO}$ is the main producer of $\mathrm{H}_{2} \mathrm{O}_{2}$ inside peroxisomes (see Introduction). Patients with specific deficiencies of peroxisomal FAO enzymes may have compensatory increased levels of ACOX, with increased ACOX-to-Catalase ratio and suffer from chronically increased oxidative stress (Ferdinandusse et al. 2002). We postulated that LPS may induce an analogous imbalance between metabolic (pro-oxidative) and anti-oxidative mechanisms inside peroxisomes. Immunoblot revealed increased of total ACOX protein in kidneys from lys-mice compared to wtmice. Analysis of ACOX enzymatic activity using palmitic acid in peroxisome-enriched kidney fraction revealed that lys-mice had initially higher activity of ACOX after 9 hours and later decrease after 24 hours following LPS injection compared to wt-mice. Consistent with ACOX enzymatic activity, levels of unmetabolized free fatty acids were lower after 9 hours and increased after 24 hours in LPS-trated lys-mice. In wt-mice, LPS-induced temporary decrease of peroxisomes was accompanied by an equal reduction of $\mathrm{H}_{2} \mathrm{O}_{2}$-generating $\mathrm{ACOX}(\mathrm{FAO})$ and $\mathrm{H}_{2} \mathrm{O}_{2}$-decomposing catalase, preserving the redox balance. Contrary, LPS-treated lys-mice showed a relative preserved ACOX (thus functional FAO) and at the same time dysfunctional catalase, resulting in redox imbalance and oxidative injury. Lysosome-competent cells showed an equal reduction of peroxisomal metabolic and antioxidative mechanisms after LPS, keeping the redox balance preserved (Figure 7). Lysosome-defective cells have higher amount of catalase protein, though with reduced enzymatic activity. During LPS exposure, they exhibited dysbalance between relatively preserved FAO and inhibited catalase producing redox dysequilibrium. The enzymatic activity of ACOX correlated with transcription activity of PPAR $\alpha$, repressed in wt- and abnormally increased in lys-mice during LPS exposure. The exact endogen stimulator of abnormally active PPAR $\alpha$ in impaired lysosomal autophagy remains obscure. PPAR $\alpha$ 
upregulation may reflect a compensatory mechanism to activate dysfunctional peroxisomes, which in case of ACOX/catalase dysbalance yields an opposite effect.

Patients with peroxisomal bifunctional protein deficiency show increased PPAR $\alpha$ and ACOX activity, a higher ACOX/catalase ratio and increased oxidative stress (Ferdinandusse et al. 2003). Contrary, severe peroxisomal deficiency caused by deletion of Pex 5 also activated PPAR $\alpha$, though because of practically complete lack of ACOX, did not lead to increase oxidative stress (Dirkx et al. 2005). 


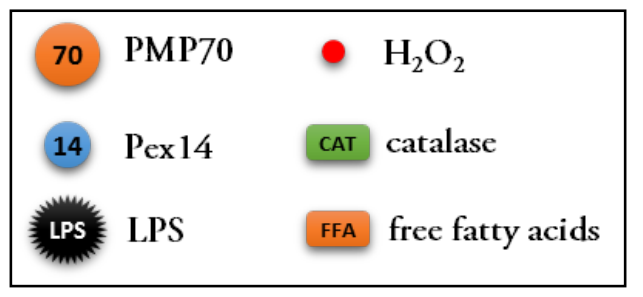

Figure 7. Schematic representation of metabolic-oxidative changes in lysosome-competent cells (A-C) and in cells with lysosomal dysfunction (D-F) after LPS exposure. (A-C) Lysosomecompetent cells. Under normal conditions, LPS activates pexophagy and induces a biphasic expression response, with an early decrease followed by upregulation of PMP70/Pex14, and increased proliferation of peroxisomes. Both, $\mathrm{H}_{2} \mathrm{O}_{2}$-generating $\mathrm{ACOX}$ and $\mathrm{H}_{2} \mathrm{O}_{2}$-decomposing catalase are decreased, thus preserving peroxisomal redox equilibrium. (D-F) Lysosomal dysfunction inhibits pexophagy, which results in accumulation of peroxisomes and higher levels of PMP70/Pex14 under basal conditions. LPS stimulation of dysfunctional peroxisomes with initially preserved ACOX activity and inhibited catalase generates $\mathrm{H}_{2} \mathrm{O}_{2}$ disequilibrium and enhances oxidative stress. Later, this metabolic-oxidative imbalance causes a global deterioration of peroxisomal homeostasis - peroxisomal burnout. 


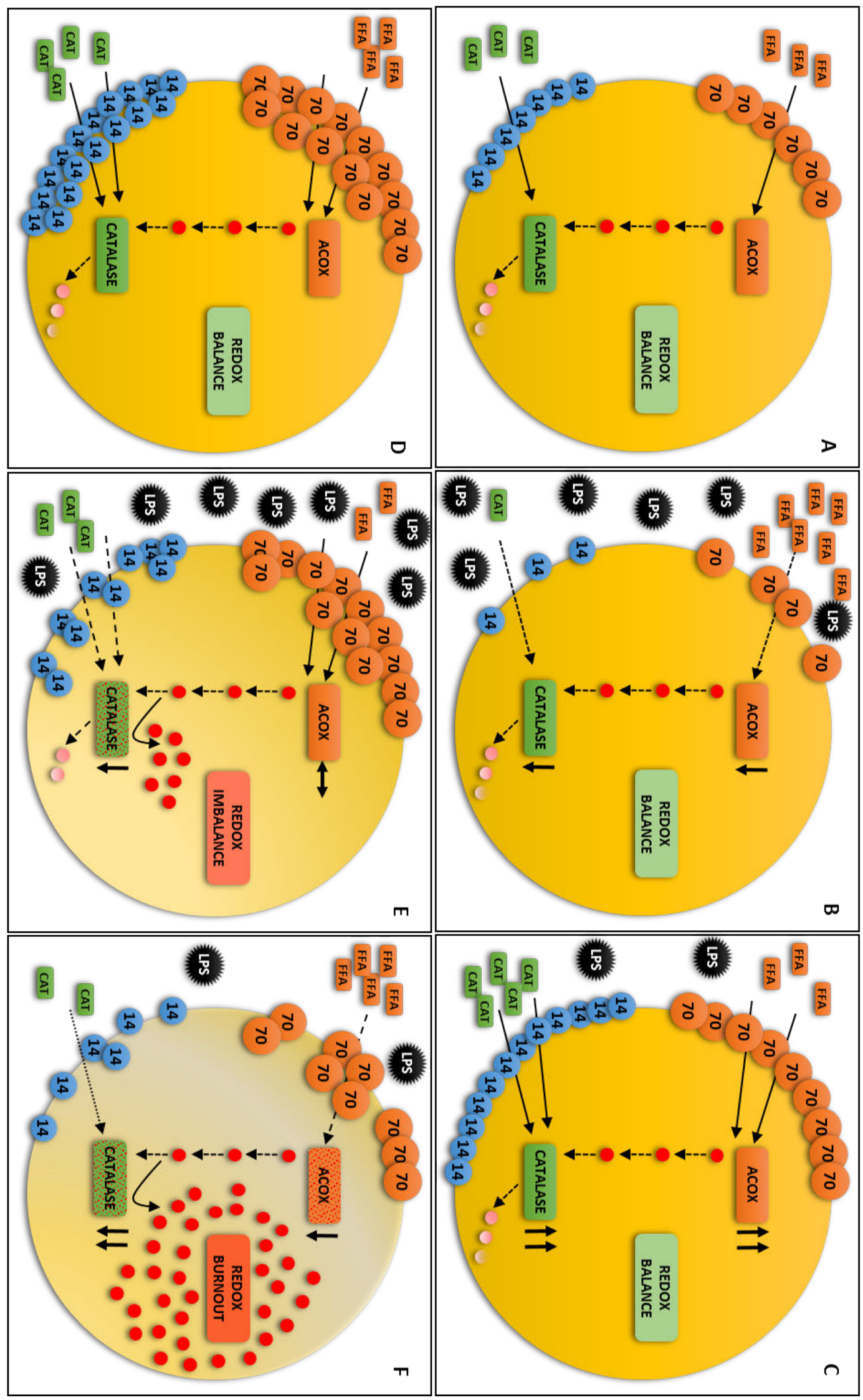




\section{Discussion}

My dissertation has it main focus on the regulation of mammalian peroxisomes during endotoxic stress in the settings of acute kidney injury. Two main objectives of the presented were (I) how LPS affects dynamics and functions of peroxisomes, and (II) how a superimposed dysfunction of lysosomes influence the default responses of peroxisomes to LPS stress

Historically, following the discovery of rather rare, genetically transmitted peroxisomal diseases in humans, these almost forgotten organelles caught again the interest of the scientific community. Today, peroxisomes have proven their position and play a prominent role in cellular (patho)physiology. We present that not only disorders of peroxisomal biogenesis, but also conditions interfering with peroxisomal degradation, particularly impaired pexophagy may contribute to the development and progression of organ damage.

The models used in this work rely on endotoxin, as a powerful stressor deeply forcing cellular oxidative and metabolic stress response pathways. The cytokine storm released by endothelial cells after stimulation with endotoxin hits peroxisomes, and as a consequence, a fraction of them get inevitably injured, develop redox imbalance and become dangerous for the cell. In the presence of competent lysosomes, LPS-induced pexophagy eliminates and recycles injured organelles, and helps to maintain a functional peroxisomal pool. Impaired pexophagy hinders turnover of peroxisomes and increases the number of organelles with disturbed redox equilibrium and excess of $\mathrm{H}_{2} \mathrm{O}_{2}$, mainly due to imbalance between preserved pro-oxidative FAO and inhibited antioxidative catalase. Additionally, autophagy dysfunction generates a continual low-grade inflammatory state. The bidirectional relationship between oxidative stress and inflammation is 
well established. The pathways connecting inflammatory and autophagy networks contain hundreds of proteins, which are focus of current research (Levine et al. 2011). One of these, the p62/SQSTM1 protein with a specific role in pexophagy is a marker protein of the autophagy flux, and accumulates, therefore, in cells with lysosomal dysfunction. High levels of accumulated p62/SQSTM1 can activate NF-kB pathway and further promote inflammation (Moscat and Diaz-Meco 2009). Lysosomes are rich in redox-active iron and sensitive to oxidative stress (Kurz et al. 2007). The progressive worsening of peroxisomal redox homeostasis in lysosomal dysfunction during endotoxin stress results in leakage of excessive $\mathrm{H}_{2} \mathrm{O}_{2}$ into cytoplasm and lysosomes. This additionally impairs lysosomal functions and creates a vicious circle scenario resulting in fatal metabolic burnout of organelles. This work demonstrated for the first time that these metabolic-oxidative "mismatch patterns" observed in patients with hereditary peroxisomal diseases are recapitulated in the molecular pathogenesis of acquired peroxisomal disorders.

When moving from the organelle to the animal, lysosome-impaired lys-mice have increased serum cytokine levels along with slight proteinuria under normal conditions, indicating an early renal impairment in these animals. Lysosomal mice treated with LPS show significantly higher serum cytokine concentrations, proteinuria and an accelerated decline of renal function. Detailed examination of renal parenchyma in endotoxin-stressed lys-mice revealed accumulation of damaged peroxisomes, dysfunctional catalase, and increased oxidative stress with excess of $\mathrm{H}_{2} \mathrm{O}_{2}$. Due to the relative preserved FAO with declined catalase activity, an initial oxidative imbalance occurs. However, in later stages, peroxisomal burnout with accumulation of cytotoxic unmetabolized fatty acids and lipid peroxidation products prevails. 
As already mentioned, the prognostic significance of functional FAO for the outcome of AKI is well-recognized (see Section 1.6. Peroxisomes and kidney). Preserved FAO is required to meet the increased energy demand of stressed kidney. In this regard, we would complement this statement by reinforcing the importance of preserved autophagy/pexophagy in prevention of functional disconnection between metabolic and oxidative pathways within cells.

Magnetic resonance studies demonstrated abnormalities in kidney parenchyma as early as six hours after septic insult (Dear et al. 2005). This corresponds well to our findings on molecular level demonstrating increased renal concentrations of malondialdehyde, a product of lipid peroxidation already 9 hours after LPS injection. This occurs even before conventional serum and urinary markers of renal dysfunction become abnormal. This emphasizes the relevance of early subclinical alterations, offering potential diagnostic and therapeutic targets in inflammatory kidney disease.

One of the therapeutic approaches derived from biochemical, and particularly lipid metabolic alterations detected in renal parenchyma in AKI has been the stimulation of PPAR $\alpha$, the principal activator of peroxisomes. Contrary to repressed PPAR $\alpha$ in wt-mice, we noticed an abnormally activated PPAR $\alpha$ (with subsequent FAO/catalase imbalance) in lysosomal mice during LPS stress. Here, as well, the statement on the protective role of PPAR $\alpha$ in AKI needs to be complemented by "under conditions with preserved autophagy/pexophagy". Studies demonstrating protective effects of PPAR $\alpha$ stimulation in early diabetic nephropathy were unable to reproduce these effects in progressive disease (Las and Shirihai 2010). Lysosomal dysfunction is a common companion of chronic diseases, such as diabetes, and might have contributed to this inconsistency observed at an advanced disease stage (Boya 2012). 
Mounting evidence suggests that peroxisomal dysfunction per se may be implicated in pathogenesis of aging and age-related diseases, like neurodegeneration, type 2 diabetes or even cancer. Peroxisomes function as a source, sink, or target of ROS and may also act as a signaling platform for a variety of age-related processes (Fransen et al. 2013).

Our model of endotoxin-induced AKI is an extreme example of deleterious consequences of impaired lysosomal function for the cellular homeostasis. We propose that mechanisms described in these acute settings may participate in the pathogenesis of common human chronic diseases, though at lower intensities and in longer period of time.

There is emerging evidence that subclinical circulating levels of endotoxin, released by bacterial flora of the digestive system are associated with increased cardiovascular burden, particularly in patients with chronic kidney disease (McIntyre et al. 2011). Association between the circulating endotoxin levels and the degree of renal impairment was also reported by Szeto (Szeto et al. 2008). In this respect, our study is first to offer a mechanistic link to peroxisome as one of the culprits in the pathogenesis of chronic kidney diseases.

Lysosomal defects have impact on virtually all cellular compartments and we realize that pexophagy explains only a fraction of the findings, and is a part of the responses to endotoxic stress. Nonetheless, major conclusions obtained here in animal studies were buttressed by the results obtained in cells, thus minimizing over-interpretation of the data.

In conclusion, our data strongly suggest that activated pexophagy, a physiological cellular mechanism per se, plays a fundamental role in quality selection, degradation and renewal of peroxisomes during endotoxic stress. We demonstrated that pexophagy is a default response to 
LPS. Pexophagy refreshes peroxisomal pool and is essential for various cellular functions, particularly during cellular stress. Conversely, when lysosomal dysfunction is superimposed, impairment of pexophagy results in accumulation of compromised peroxisomes with severe redox imbalance that contributes significantly to increased cellular oxidative stress and aggravated kidney damage.

Although the discovery of peroxisomes celebrate its $60^{\text {th }}$ anniversary, the contribution of peroxisomes to the pathogenesis of common human diseases remains still scarcely understood at present. With this dissertation, the doctoral candidate hopes to contribute to further research on this organelle aimed at identifying potential targets for novel peroxisome-directed diagnostic and therapeutic interventions. 


\section{References}

Andonegui G, Zhou H, Bullard D, Kelly MM, Mullaly SC, McDonald B, Long EM, Robbins SM and Kubes P (2009): Mice that exclusively express TLR4 on endothelial cells can efficiently clear a lethal systemic Gram-negative bacterial infection. J Clin Invest 119(7): 1921-1930

Baes M and Van Veldhoven PP (2012): Mouse models for peroxisome biogenesis defects and beta-oxidation enzyme deficiencies. Biochim Biophys Acta 1822(9): 1489-1500

Bagattin A, Hugendubler L and Mueller E (2010): Transcriptional coactivator PGC-1alpha promotes peroxisomal remodeling and biogenesis. Proc Natl Acad Sci U S A 107(47): 2037620381

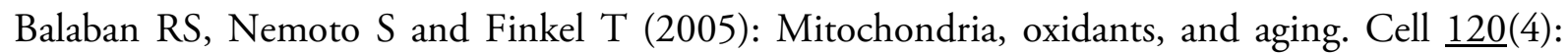
$483-495$

Behrends C and Fulda S (2012): Receptor proteins in selective autophagy. Int J Cell Biol 2012: 673290

Bharti P, Schliebs W, Schievelbusch T, Neuhaus A, David C, Kock K, Herrmann C, Meyer HE, Wiese S, Warscheid B, Theiss C and Erdmann R (2011): PEX14 is required for microtubulebased peroxisome motility in human cells. J Cell Sci 124(Pt 10): 1759-1768

Bonekamp NA, Volkl A, Fahimi HD and Schrader M (2009): Reactive oxygen species and peroxisomes: struggling for balance. Biofactors $\underline{35}(4)$ : 346-355

Borradaile NM and Pickering JG (2009): NAD(+), sirtuins, and cardiovascular disease. Curr Pharm Des 15(1): 110-117

Boya P (2012): Lysosomal function and dysfunction: mechanism and disease. Antioxid Redox Signal 17(5): 766-774

Braverman NE, D'Agostino MD and Maclean GE (2013): Peroxisome biogenesis disorders: Biological, clinical and pathophysiological perspectives. Dev Disabil Res Rev 17(3): 187-196

Brink SC and Danpure CJ (2001): Protein Import into Peroxisomes: Methods. eLS 
Cunningham PN, Dyanov HM, Park P, Wang J, Newell KA and Quigg RJ (2002): Acute renal failure in endotoxemia is caused by TNF acting directly on TNF receptor-1 in kidney. J Immunol 168(11): 5817-5823

Cunningham PN, Wang Y, Guo R, He G and Quigg RJ (2004): Role of Toll-like receptor 4 in endotoxin-induced acute renal failure. J Immunol 172(4): 2629-2635

De Duve C (2007): The origin of eukaryotes: a reappraisal. Nat Rev Genet $\underline{8}(5)$ : 395-403

De Duve C and Baudhuin P (1966): Peroxisomes (microbodies and related particles). Physiol $\operatorname{Rev} \underline{46}(2): 323-357$

Dear JW, Kobayashi H, Jo SK, Holly MK, Hu X, Yuen PS, Brechbiel MW and Star RA (2005): Dendrimer-enhanced MRI as a diagnostic and prognostic biomarker of sepsis-induced acute renal failure in aged mice. Kidney Int $\underline{67(6): 2159-2167}$

Dirkx R, Vanhorebeek I, Martens K, Schad A, Grabenbauer M, Fahimi D, Declercq P, Van Veldhoven PP and Baes M (2005): Absence of peroxisomes in mouse hepatocytes causes mitochondrial and ER abnormalities. Hepatology $\underline{41}(4): 868-878$

Dixit E, Boulant S, Zhang Y, Lee AS, Odendall C, Shum B, Hacohen N, Chen ZJ, Whelan SP, Fransen M, Nibert ML, Superti-Furga G and Kagan JC (2010): Peroxisomes are signaling platforms for antiviral innate immunity. Cell $\underline{141(4): 668-681}$

Eadon MT, Hack BK, Xu C, Ko B, Toback FG and Cunningham PN (2012): Endotoxemia alters tight junction gene and protein expression in the kidney. Am J Physiol Renal Physiol 303(6): F821-830

Feldkamp T, Kribben A, Roeser NF, Senter RA and Weinberg JM (2006): Accumulation of nonesterified fatty acids causes the sustained energetic deficit in kidney proximal tubules after hypoxia-reoxygenation. Am J Physiol Renal Physiol 290(2): F465-477

Ferdinandusse S, Finckh B, de Hingh YC, Stroomer LE, Denis S, Kohlschutter A and Wanders RJ (2003): Evidence for increased oxidative stress in peroxisomal D-bifunctional protein deficiency. Mol Genet Metab $\underline{79}(4):$ 281-287 
Ferdinandusse S, Meissner T, Wanders RJ and Mayatepek E (2002): Identification of the peroxisomal beta-oxidation enzymes involved in the degradation of leukotrienes. Biochem Biophys Res Commun 293(1): 269-273

Fransen M, Nordgren M, Wang B, Apanasets O and Van Veldhoven PP (2013): Aging, agerelated diseases and peroxisomes. Subcell Biochem 69: 45-65

Gabaldon T (2010): Peroxisome diversity and evolution. Philos Trans R Soc Lond B Biol Sci $\underline{365(1541): 765-773}$

Gulati S, Ainol L, Orak J, Singh AK and Singh I (1993): Alterations of peroxisomal function in ischemia-reperfusion injury of rat kidney. Biochim Biophys Acta 1182(3): 291-298

Gulati S, Singh AK, Irazu C, Orak J, Rajagopalan PR, Fitts CT and Singh I (1992): Ischemiareperfusion injury: biochemical alterations in peroxisomes of rat kidney. Arch Biochem Biophys 295(1): 90-100

Hara-Kuge S and Fujiki Y (2008): The peroxin Pex14p is involved in LC3-dependent degradation of mammalian peroxisomes. Exp Cell Res 314(19): 3531-3541

Harding M and Kubes P (2012): Innate immunity in the vasculature: interactions with pathogenic bacteria. Curr Opin Microbiol 15(1): 85-91

Hasegawa K, Wakino S, Yoshioka K, Tatematsu S, Hara Y, Minakuchi H, Sueyasu K, Washida N, Tokuyama H, Tzukerman M, Skorecki K, Hayashi K and Itoh H (2010): Kidney-specific overexpression of Sirt1 protects against acute kidney injury by retaining peroxisome function. J Biol Chem 285(17): 13045-13056

Hashimoto T (1996): Peroxisomal beta-oxidation: enzymology and molecular biology. Ann N Y Acad Sci 804: 86-98

Hess R, Staubli W and Riess W (1965): Nature of the hepatomegalic effect produced by ethylchlorophenoxy-isobutyrate in the rat. Nature 208(5013): 856-858

Huang Q, Yeldandi A, Alvares K, Ide H, Reddy J and Rao M (1995): Localization of peroxisome proliferator-activated receptor in mouse and rat-tissues and demonstration of its nuclear translocation in transfected cv-1 cells. Int J Oncol $\underline{6}(2): 307-312$ 
Hunt MC and Alexson SE (2008): Novel functions of acyl-CoA thioesterases and acyltransferases

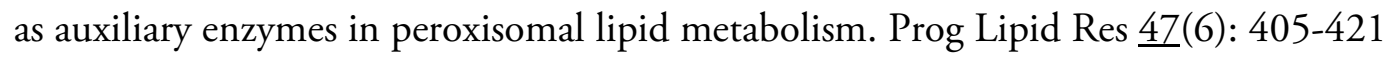

Huybrechts SJ, Van Veldhoven PP, Brees C, Mannaerts GP, Los GV and Fransen M (2009): Peroxisome dynamics in cultured mammalian cells. Traffic 10(11): 1722-1733

Hwang I, Lee J, Huh JY, Park J, Lee HB, Ho YS and Ha H (2012): Catalase deficiency accelerates diabetic renal injury through peroxisomal dysfunction. Diabetes $\underline{61}(3)$ : 728-738

Inestrosa NC, Carvajal FJ, Zolezzi JM, Tapia-Rojas C, Serrano F, Karmelic D, Toledo EM, Toro A, Toro J and Santos MJ (2013): Peroxisome proliferators reduce spatial memory impairment, synaptic failure, and neurodegeneration in brains of a double transgenic mice model of Alzheimer's disease. J Alzheimers Dis $\underline{33}$ (4): 941-959

Islinger M, Grille S, Fahimi HD and Schrader M (2012): The peroxisome: an update on mysteries. Histochem Cell Biol 137(5): 547-574

Issemann I and Green S (1990): Activation of a member of the steroid hormone receptor superfamily by peroxisome proliferators. Nature 347(6294): 645-650

Ivashchenko O, Van Veldhoven PP, Brees C, Ho YS, Terlecky SR and Fransen M (2011): Intraperoxisomal redox balance in mammalian cells: oxidative stress and interorganellar cross-talk. Mol Biol Cell 22(9): 1440-1451

Kim PK, Hailey DW, Mullen RT and Lippincott-Schwartz J (2008): Ubiquitin signals autophagic degradation of cytosolic proteins and peroxisomes. Proc Natl Acad Sci U S A 105(52): 20567-20574

Koepke JI, Nakrieko KA, Wood CS, Boucher KK, Terlecky LJ, Walton PA and Terlecky SR (2007): Restoration of peroxisomal catalase import in a model of human cellular aging. Traffic $\underline{8}(11): 1590-1600$

Kurz T, Terman A and Brunk UT (2007): Autophagy, ageing and apoptosis: the role of oxidative stress and lysosomal iron. Arch Biochem Biophys $\underline{462(2): ~ 220-230 ~}$

Las G and Shirihai OS (2010): The role of autophagy in beta-cell lipotoxicity and type 2 diabetes. Diabetes Obes Metab 12 Suppl 2: 15-19 
Lazarow PB and Fujiki Y (1985): Biogenesis of peroxisomes. Annu Rev Cell Biol 11: 489-530

Le Hir M and Dubach UC (1982): Peroxisomal and mitochondrial beta-oxidation in the rat kidney: distribution of fatty acyl-coenzyme A oxidase and 3-hydroxyacyl-coenzyme A dehydrogenase activities along the nephron. J Histochem Cytochem 30(5): 441-444

Lee SS, Pineau T, Drago J, Lee EJ, Owens JW, Kroetz DL, Fernandez-Salguero PM, Westphal H and Gonzalez FJ (1995): Targeted disruption of the alpha isoform of the peroxisome proliferatoractivated receptor gene in mice results in abolishment of the pleiotropic effects of peroxisome proliferators. Mol Cell Biol 15(6): 3012-3022

Legakis JE, Koepke JI, Jedeszko C, Barlaskar F, Terlecky LJ, Edwards HJ, Walton PA and Terlecky SR (2002): Peroxisome senescence in human fibroblasts. Mol Biol Cell 13(12): 42434255

Levine B, Mizushima N and Virgin HW (2011): Autophagy in immunity and inflammation. Nature $\underline{469}$ (7330): 323-335

Li S, Mariappan N, Megyesi J, Shank B, Kannan K, Theus S, Price PM, Duffield JS and Portilla D (2013): Proximal tubule PPARalpha attenuates renal fibrosis and inflammation caused by unilateral ureteral obstruction. Am J Physiol Renal Physiol 305(5): F618-627

Li S, Nagothu KK, Desai V, Lee T, Branham W, Moland C, Megyesi JK, Crew MD and Portilla D (2009): Transgenic expression of proximal tubule peroxisome proliferator-activated receptoralpha in mice confers protection during acute kidney injury. Kidney Int $\underline{76}(10)$ : 1049-1062

Li S, Wu P, Yarlagadda P, Vadjunec NM, Proia AD, Harris RA and Portilla D (2004): PPAR alpha ligand protects during cisplatin-induced acute renal failure by preventing inhibition of renal FAO and PDC activity. Am J Physiol Renal Physiol 286(3): F572-580

Ma C, Agrawal G and Subramani S (2011): Peroxisome assembly: matrix and membrane protein biogenesis. J Cell Biol 193(1): 7-16

Ma C, Schumann U, Rayapuram N and Subramani S (2009): The peroxisomal matrix import of Pex8p requires only PTS receptors and Pex14p. Mol Biol Cell 20(16): 3680-3689

Mannaerts GP and Van Veldhoven PP (1993): [Peroxisomal beta-oxidation]. Verh K Acad Geneeskd Belg 55(1): 45-78 
Masters CJ (1996): Cellular signalling: the role of the peroxisome. Cell Signal $\underline{8}(3): 197-208$

McIntyre CW, Harrison LE, Eldehni MT, Jefferies HJ, Szeto CC, John SG, Sigrist MK, Burton JO, Hothi D, Korsheed S, Owen PJ, Lai KB and Li PK (2011): Circulating endotoxemia: a novel factor in systemic inflammation and cardiovascular disease in chronic kidney disease. Clin J Am Soc Nephrol $\underline{6}(1): 133-141$

Melican K, Boekel J, Mansson LE, Sandoval RM, Tanner GA, Kallskog O, Palm F, Molitoris BA and Richter-Dahlfors A (2008): Bacterial infection-mediated mucosal signalling induces local renal ischaemia as a defence against sepsis. Cell Microbiol 10(10): 1987-1998

Mizushima N and Komatsu M (2011): Autophagy: renovation of cells and tissues. Cell 147(4): 728-741

Moscat J and Diaz-Meco MT (2009): p62 at the crossroads of autophagy, apoptosis, and cancer. Cell 137(6): 1001-1004

Nuttall JM, Motley A and Hettema EH (2011): Peroxisome biogenesis: recent advances. Curr Opin Cell Biol 23(4): 421-426

Odendall C and Kagan JC (2013): Peroxisomes and the antiviral responses of Mammalian cells. Subcell Biochem 69: 67-75

Platta HW and Erdmann R (2007): Peroxisomal dynamics. Trends Cell Biol 17(10): 474-484

Portilla D (1999): Role of fatty acid beta-oxidation and calcium-independent phospholipase A2 in ischemic acute renal failure. Curr Opin Nephrol Hypertens $\underline{8}(4): 473-477$

Portilla D, Dai G, McClure T, Bates L, Kurten R, Megyesi J, Price P and Li S (2002): Alterations of PPARalpha and its coactivator PGC-1 in cisplatin-induced acute renal failure. Kidney Int $\underline{62}(4): 1208-1218$

Purdue PE and Lazarow PB (1996): Targeting of human catalase to peroxisomes is dependent upon a novel COOH-terminal peroxisomal targeting sequence. J Cell Biol 134(4): 849-862

Purdue PE and Lazarow PB (2001): Peroxisome biogenesis. Annu Rev Cell Dev Biol 17: 701-752 
Ravikumar B, Berger Z, Vacher C, O'Kane CJ and Rubinsztein DC (2006): Rapamycin pretreatment protects against apoptosis. Hum Mol Genet 15(7): 1209-1216

Reddy JK and Hashimoto T (2001): Peroxisomal beta-oxidation and peroxisome proliferatoractivated receptor alpha: an adaptive metabolic system. Annu Rev Nutr 21: 193-230

Rhodin J (1954): Correlation of ultrastructural organization and function in normal experimentally changed convoluted tubule cells of the mouse kidney. Thesis. Karolinska Institutet, Stockholm,

Rivera-Zavala JB, Baez-Ruiz A and Diaz-Munoz M (2011): Changes in the 24 h Rhythmicity of Liver PPARs and Peroxisomal Markers When Feeding Is Restricted to Two Daytime Hours. PPAR Res 2011: 261584

Rottensteiner H, Kramer A, Lorenzen S, Stein K, Landgraf C, Volkmer-Engert R and Erdmann R (2004): Peroxisomal membrane proteins contain common Pex19p-binding sites that are an integral part of their targeting signals. Mol Biol Cell 15(7): 3406-3417

Rucktaschel R, Girzalsky W and Erdmann R (2011): Protein import machineries of peroxisomes. Biochim Biophys Acta 1808(3): 892-900

Ruidera E, Irazu CE, Rajagopalan PR, Orak JK, Fitts CT and Singh I (1988): Fatty acid metabolism in renal ischemia. Lipids 23(9): 882-884

Scherz-Shouval R, Shvets E, Fass E, Shorer H, Gil L and Elazar Z (2007): Reactive oxygen species are essential for autophagy and specifically regulate the activity of Atg4. EMBO J 26 (7): $1749-1760$

Schluter A, Fourcade S, Ripp R, Mandel JL, Poch O and Pujol A (2006): The evolutionary origin of peroxisomes: an ER-peroxisome connection. Mol Biol Evol 23(4): 838-845

Schrader M, Bonekamp NA and Islinger M (2012): Fission and proliferation of peroxisomes. Biochim Biophys Acta 1822(9): 1343-1357

Sigfrid LA, Cunningham JM, Beeharry N, Lortz S, Tiedge M, Lenzen S, Carlsson C and Green IC (2003): Cytokines and nitric oxide inhibit the enzyme activity of catalase but not its protein or mRNA expression in insulin-producing cells. J Mol Endocrinol 31(3): 509-518 
Smith JJ and Aitchison JD (2013): Peroxisomes take shape. Nat Rev Mol Cell Biol 144(12): 803817

Steinberg BE, Goldenberg NM and Lee WL (2012): Do viral infections mimic bacterial sepsis? The role of microvascular permeability: A review of mechanisms and methods. Antiviral Res 93(1): 2-15

Szeto CC, Kwan BC, Chow KM, Lai KB, Chung KY, Leung CB and Li PK (2008): Endotoxemia is related to systemic inflammation and atherosclerosis in peritoneal dialysis patients. Clin J Am Soc Nephrol $\underline{3}(2)$ : 431-436

Teijaro JR, Walsh KB, Cahalan S, Fremgen DM, Roberts E, Scott F, Martinborough E, Peach R, Oldstone MB and Rosen $\mathrm{H}$ (2011): Endothelial cells are central orchestrators of cytokine amplification during influenza virus infection. Cell 146(6): 980-991

Terman A and Kurz T (2013): Lysosomal iron, iron chelation, and cell death. Antioxid Redox Signal $\underline{18}(8): 888-898$

Till A, Lakhani R, Burnett SF and Subramani S (2012): Pexophagy: the selective degradation of peroxisomes. Int J Cell Biol 2012: 512721

Todde V, Veenhuis M and van der Klei IJ (2009): Autophagy: principles and significance in health and disease. Biochim Biophys Acta 1792(1): 3-13

Van Veldhoven PP and Baes M (2013): Peroxisome deficient invertebrate and vertebrate animal models. Front Physiol ㄸ: 335

Vasko R, Xavier S, Chen J, Lin CH, Ratliff B, Rabadi M, Maizel J, Tanokuchi R, Zhang F, Cao J and Goligorsky MS (2014): Endothelial sirtuin 1 deficiency perpetrates nephrosclerosis through downregulation of matrix metalloproteinase-14: relevance to fibrosis of vascular senescence. J Am Soc Nephrol 25(2): 276-291

Wanders RJ, Visser WF, van Roermund CW, Kemp S and Waterham HR (2007): The peroxisomal ABC transporter family. Pflugers Arch $\underline{453}(5): 719-734$

Wu L, Tiwari MM, Messer KJ, Holthoff JH, Gokden N, Brock RW and Mayeux PR (2007): Peritubular capillary dysfunction and renal tubular epithelial cell stress following lipopolysaccharide administration in mice. Am J Physiol Renal Physiol 292(1): F261-268 
Yakunin E, Moser A, Loeb V, Saada A, Faust P, Crane DI, Baes M and Sharon R (2010): alphaSynuclein abnormalities in mouse models of peroxisome biogenesis disorders. J Neurosci Res $\underline{88}(4): 866-876$

Yokota S and Dariush Fahimi H (2009): Degradation of excess peroxisomes in mammalian liver cells by autophagy and other mechanisms. Histochem Cell Biol 131(4): 455-458

Yokota S, Oda T and Fahimi HD (2001): The role of 15-lipoxygenase in disruption of the peroxisomal membrane and in programmed degradation of peroxisomes in normal rat liver. $\mathrm{J}$ Histochem Cytochem $\underline{\text { 49(5): 613-622 }}$

Zmijewski JW, Lorne E, Zhao X, Tsuruta Y, Sha Y, Liu G and Abraham E (2009): Antiinflammatory effects of hydrogen peroxide in neutrophil activation and acute lung injury. Am J Respir Crit Care Med 179(8): 694-704 


\section{Thesis publication}

Vasko R, Ratliff BB, Bohr S, Nadel E, Chen J, Xavier S, Chander P, Goligorsky MS (2013):

Endothelial Peroxisomal Dysfunction and Impaired Pexophagy Promotes Oxidative Damage in Lipopolysaccharide-Induced Acute Kidney Injury. Antioxid Redox Signal 19(3):211-230.

http://www.ncbi.nlm.nih.gov/pubmed/23088293 